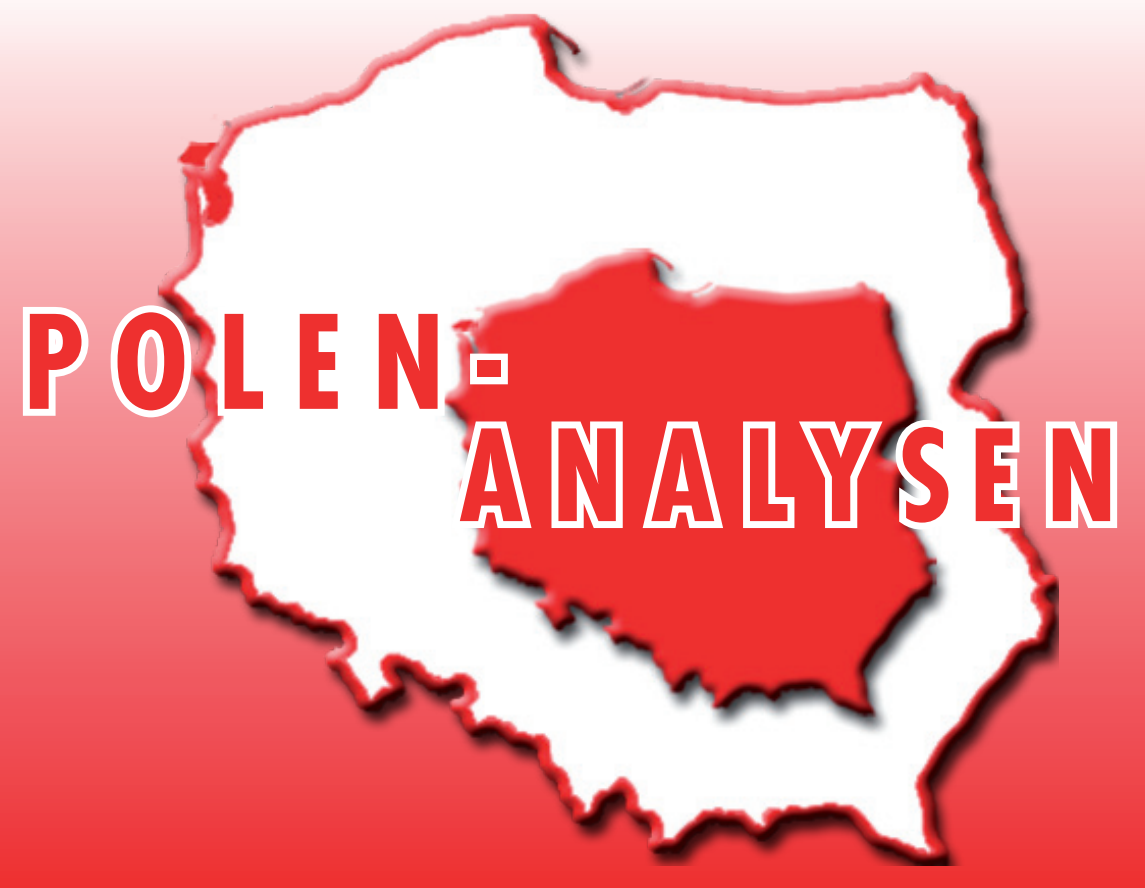

www.laender-analysen.de/polen

\title{
POLEN, DER UKRAINE-KONFLIKT UND DIE EUROPÄISCHE UNION
}

ANALYSE

Polen, der Ukraine-Konflikt und die Europäische Union

Piotr Buras, Warschau

- TABELLEN UND GRAFIKEN ZUM TEXT

Umfragen zum Ukraine-Konflikt und zum Verhältnis gegenüber anderen Nationen

- CHRONIK

17. März 2015 - 20. April 2015 


\title{
Polen, der Ukraine-Konflikt und die Europäische Union
}

\author{
Piotr Buras, Warschau
}

\section{Zusammenfassung}

Die neue Regierung von Ministerpräsidentin Ewa Kopacz, die im Herbst nach der Wahl Donald Tusks zum Präsidenten des Europäischen Rates gebildet wurde, setzte keine neuen Akzente in der Politik zum UkraineKonflikt. In der Frage der Unterstützung der Ukraine bemüht sich Warschau, nicht aus der Argumentationslinie auszuscheren, dass die Einheit der Europäischen Union und des Westens wichtiger sei als Aktionismus. Polen war auch nicht bei den Hauptverhandlungen mit Russland dabei. Polen hat nur beschränkte Möglichkeiten, auf die Lösung des russisch-ukrainischen Konflikts Einfluss zu nehmen, legt aber Nachdruck auf die Stärkung der NATO und der EU sowie der eigenen Verteidigungsfähigkeit.

$\mathrm{D}$ Regierungswechsel infolge der Wahl Donald Tusks zum Präsidenten des Europäischen Rates und der Abgang des Duetts Donald Tusk - Radosław Sikorski, das die polnische Außenpolitik sieben Jahre gestaltet hatte, traf die polnische Diplomatie in einem besonders schwierigen Moment. Der russisch-ukrainische Konflikt hatte nicht nur zur Folge, dass in der unmittelbaren Nachbarschaft Polens erstzunehmende Bedrohungen für die Sicherheit des Staates auftraten. Er offenbarte auch die Beschränkungen der polnischen Diplomatie und ihrer Fähigkeiten, auf die Politik der Europäischen Union Einfluss zu nehmen. Zwar hatte Warschau in der ersten Phase der Krise, Anfang 2014, nicht nur in den Verhandlungen in Kiew (gemeinsam mit Deutschland und Frankreich) eine Schlüsselrolle gespielt, sondern auch durch die unnachgiebige Mobilisierung der EU-Partner zugunsten entschiedenerer Aktivitäten angesichts des russischen Vorgehens gegenüber der Ukraine. Dessen ungeachtet wurde die Rolle Polens im Laufe der Zeit schwächer, was seinen symbolischen Ausdruck im sogenannten Normandie-Format fand, den im Juni 2014 initiierten Gesprächen zwischen den Vertretern Deutschlands, Frankreichs, Russlands und der Ukraine. Diese wurden das Hauptdiskussionsforum über die Lösungsmöglichkeiten des Konfliktes - ohne Beteiligung Polens, trotz seiner offenkundigen Bemühungen, diesem Kreis anzugehören.

Dass Warschau nicht eingeladen worden war, war im Sommer 2014 Gegenstand scharfer Kritik in den Medien, an der sich auch Politiker und Anhänger der Regierungskoalition beteiligten. Sie zielte vor allem auf Deutschland, das als Architekt der europäischen Politik und daher als verantwortlich für das Gesprächsformat mit Moskau galt. Dieses käme, so die Argumentation, den russischen Erwartungen entgegen, da es diejenigen Akteure ausschloss, die Russland gegenüber kritischer eingestellt seien als Berlin und Paris. Darüber, dass die Politik Deutschlands »eine Bombe unter dem Fundament Europas« sei und dass "man sich nicht auf Deutsch- land verlassen « könne, schrieben renommierte polnische Politiker und Kommentatoren. Der Ton dieser Diskussion wurde zusätzlich durch polnisch-deutsche Diskrepanzen vor dem Hintergrund der Entscheidungen des NATO-Gipfels in Newport im September 2014 verschärft. Während sich Warschau u. a. für die Stärkung der Ostflanke der NATO mit Hilfe der ständigen Stationierung von Truppen der Bündnispartner aussprach, wollte Berlin um jeden Preis Schritte vermeiden, die Russland allzu sehr zum Antagonisten machen könnten, beispielsweise indem die Grundsätze der NATO-Russland-Grundakte von 1997 verletzt würden (die Russland übrigens selbst vergewaltigt hatte). Die polnischdeutsche Führung in der EU-Ostpolitik stand unter einem Fragezeichen.

Im »Normandie-Format« wurde das Waffenstillstandsabkommen vom 5. September 2014 vereinbart, das jedoch keine dauerhafte Stabilisierung der Situation und Wahrung der Interessen der Ukraine zeitigte. Polen stellte diesen Prozess nicht in Frage, auch wenn in Warschau insbesondere die Verschiebung der Implementierung des Freihandelsabkommens zwischen der Europäischen Union und der Ukraine auf den 1. Januar 2016 kritisch als Geste gegenüber Moskau betrachtet wurde. Über die Fortschritte der Gespräche wurde Polen auf dem Laufenden gehalten und entgegen der zitierten Kommentare bestätigten diese nicht den Verdacht, dass versucht werden sollte, hinter dem Rücken Polens und der EU und auf Kosten der Ukraine eine Übereinkunft mit Russland zu erreichen. Die deutsche Politik gegenüber Russland, und unter dem Einfluss Berlins auch die Politik der gesamten EU, verschärfte sich deutlich im Sommer 2014, was die Verhängung der zweiten Runde von Sanktionen infolge des Abschusses eines malaysischen Flugzeugs durch prorussische Separatisten im Juli 2014 nach sich zog. In Schlüsselfragen des Konfliktes in der Ukraine wich die Haltung Polens nicht von der von Deutschland unterstützten Politik ab. Dennoch blieb das Gefühl politischer Marginalisierung in für Polen 
wichtigen außenpolitischen Bereichen. Hinzu kam, dass nicht nur die Zusammenarbeit im "Weimarer Dreieck" mit Deutschland und Frankreich, sondern auch in der Visegrád-Gruppe, deren Länder in der Frage der Ukraine andere Positionen als Polen vertraten, viel zu wünschen übrig ließ. Wichtiger ist jedoch, dass das »NormandieFormat« gegenwärtig nicht so sehr als problematisch aufgefasst wird, weil Polen keinen Platz darin hat, sondern vielmehr, weil die Vereinigten Staaten dort nicht anwesend sind. Die Festigung dieses Formats, so die Argumentation, kann dazu führen, dass es das Hauptforum für Gespräche mit Russland auch über Themen wird, die über die Fragen der Ukraine hinausgehen und die europäische Ordnung im Allgemeinen betreffen. Wenn in Schlüsselfragen der Sicherheit Europas die Vereinigten Staaten außerhalb des Entscheidungskreises blieben, würde dies einen Erfolg für Russland bedeuten, dessen Ziel es sei, die USA aus Europa zu verdrängen, sowie auch eine wichtige geopolitische Änderung auf dem Kontinent zum Nachteil der Europäischen Union.

\section{Die Einheit der EU über alles}

Die neue Ministerpräsidentin Ewa Kopacz und der neue Außenminister Grzegorz Schetyna übernahmen ihre Ämter also in einem Moment, als die polnische Politik in vielerlei Hinsicht an ihre Grenzen gestoßen war. Die Hoffnungen, dass eine neue Postenverteilung in der Regierung neue Energien und Ideen freisetzen würde - Außenminister Schetyna kündigte an, dass »der Platz Polens am Verhandlungstisch« sei, und suggerierte damit, dass sich Warschau um Anschluss an das "Normandie-Format" bemühen werde -, mischten sich mit Befürchtungen, dass die in internationalen Fragen unerfahrenen Nachfolger von Tusk und Sikorski diese Erwartungen nicht würden erfüllen können. Im Grunde zeigten die ersten Erklärungen der neuen Regierung nicht, dass Polen beabsichtige, in Sachen Ukraine mit größerer Aktivität in Erscheinung zu treten. Sowohl die Ministerpräsidentin als auch ihr Außenminister unterstrichen vor allem die Notwendigkeit, die polnische Außenpolitik im Rahmen der Europäischen Union zu koordinieren, und verabschiedeten sich davon, etwas auf eigene Faust zu unternehmen. Als Schetyna die Grundsätze der Außenpolitik im Sejm präsentierte, stellte er keine neuen Ideen für eine Strategie gegenüber dem Osten vor, sondern legte stattdessen größeren Nachdruck auf die Vertretung polnischer Interessen in außereuropäischen Ländern. Doch sollten rhetorische Unterschiede nicht den Kern der Sache verdecken: Auch das Gespann Tusk und Sikorski ließ sich von der Priorität einer gemeinsamen Linie der EU gegenüber Russland und der Ukraine leiten. Sikorski selbst hatte bereits im Jahr 2009 das Ende der »jagiellonischen Politik« verkündet, d. h. der
Politik, die Polen eine besondere Mission in Osteuropa zuschreibt, und gefordert, dass die polnische Ostpolitik der umfassenderen Vision vom Platz Polens und der polnischen Politik in der EU untergeordnet und in diesem Zusammenhang auch in die Beziehungen zu Deutschland und Frankreich eingeordnet werden sollte. In der Praxis bedeutete diese neue Philosophie nicht, dass die ambitionierte Politik gegenüber dem Osten aufgegeben wurde (was die Opposition der Regierung Tusk vorwarf), sondern vor allem die Anerkennung, dass die Wirksamkeit dieser Politik vom Platz Polens in Europa abhängt und sie nicht ein Ziel an sich ist.

Diese Einstellung wurde auch die Devise des Teams Kopacz-Schetyna. Die »Einheit der Europäischen Union « gegenüber dem Konflikt im Osten wurde spätestens seit Herbst 2014 eine Priorität der polnischen Politik, die nicht mit weitergehenden Initiativen in Eigenregie hervortrat, die grundsätzlich vom Mainstream der EU abwichen, der von Deutschland vorgegeben wurde. "Es ist besser, gemeinsam weniger zu machen, als mehr in Einzelinitiative«, sagte ein hoher polnischer Diplomat. Polen bezog auch in Fragen der Ausrüstung der ukrainischen Armee eindeutig Position. Während Anfang 2015 die Unterstützung für einen solchen Schritt in der medialen Diskussion dominierte (die wichtigsten Tageszeitungen Rzeczpospolita und Gazeta Wyborcza sprachen sich für die Nachrüstung der Ukraine aus), schloss die Regierung diese Möglichkeit aus, trotz offener Bemühungen von Seiten der Ukraine. "Die Lieferung schweren Geräts, der Ausrüstung von Spezialeinheiten, von Panzern oder ähnlicher Bewaffnung in die Ukraine kommt nicht in Frage. Polen hatte und hat keine Pläne dieser Art«, sagte Verteidigungsminister Tomasz Siemoniak in einem Interview für die Gazeta Wyborcza am 10. Februar 2015. Gleichzeitig unterstrich er, dass es anders als in Deutschland »keine Verurteilung der Zusammenarbeit der Rüstungsindustrie Polens und der Ukraine gibt. [...] Wir haben der ukrainischen Armee bereits früher geholfen und Decken und Lebensmittelrationen geschickt.» Die Haltung Polens ist in erster Linie von der Notwendigkeit einer gemeinsamen Linie mit der EU motiviert (die Mehrheit der EU-Länder ist eindeutig gegen jedwede militärische Hilfe), aber inoffiziell auch von der Angst vor der Eskalation des Konflikts, die sich am stärksten u. a. auf Polen auswirken würde, sowie von Befürchtungen hinsichtlich des Zustands der ukrainischen Armee. Während ihres Besuchs in Kiew im Januar 2015 kündigte Ministerpräsidentin Ewa Kopacz die Bereitstellung eines Kredits in Höhe von 100 Mio. Euro für die Ukraine an, der mit einer Laufzeit von zehn Jahren für den Wiederaufbau der Donbas-Region und die Unterstützung des Reform- 
prozesses vorgesehen ist (bei der Realisierung der Projekte, die aus diesem Topf finanziert werden, sollen auch polnische Firmen beteiligt werden). Während dieses Besuchs wurde außerdem eine Vereinbarung über eine Energie-Kooperation zwischen dem ukrainischen und dem polnischen Konzern Naftohaz und Gaz-System unterzeichnet, und zwar in Form eines Energietransfers für Flüssigerdgas (LNG) aus Polen in die Ukraine über Interkonnektoren mit einer Kapazität bis zu 10 Mrd. Kubikmeter jährlich - hier geht es also um anderes Gas als um das russische. Ähnlich wie Großbritannien hat Polen der Ukraine auch Hilfe bei der Schulung der ukrainischen Armee angeboten; die Übungen sollen auf polnischem Territorium stattfinden. Auch Staatspräsident Bronisław Komorowski gab bei seinem Besuch in Kiew am 10. April 2015 eine Unterstützungserklärung ab, die sowohl die Notwendigkeit umfasst, Kiew die Türen zur EU offen zu halten und auf dem Gipfel der Östlichen Partnerschaft in Riga im Mai 2015 von Seiten der EU die Visumspflicht für ukrainische Staatsbürger aufzuheben, als auch die Beteiligung Polens an einer eventuellen Friedenmission der Vereinten Nationen zur Kontrolle der Einhaltung des Waffenstillstands. Jedoch, so schrieb Rzeczpospolita sarkastisch, war dies nur ein »Besuch schöner Versprechungen", der nicht über die zuvor abgegebenen Erklärungen einer bescheidenen finanziellen Unterstützung und wenig chancenreiche Initiativen (die Mission der UN, die Aufhebung der Visumspflicht) hinausging.

\section{Ukrainemüdigkeit?}

Zweifellos wird die Krise in der Ukraine in Polen weiterhin als fundamentales Problem für die Interessen und die Sicherheit des Staates betrachtet. Nach Umfragen des Meinungsforschungsinstituts CBOS von Februar 2015 sind 67 Prozent der Polen der Ansicht, dass es sich um eine Bedrohung für Europa handelt, und 55 Prozent befürchten, dass die Krise die globale Ordnung stören wird. Die Unabhängigkeit der Ukraine und ihre Verbindung zu Europa bleiben Konstanten der polnischen Staatsräson. Sichtbarer Ausdruck dessen waren unlängst die Erklärungen hoher politischer Repräsentanten, u. a. des Senatsmarschalls Bogdan Borusewicz, in denen unterstrichen wurde, dass die Vergangenheit und damit einhergehende Streitigkeiten keine Bedeutung für die polnische Unterstützung der europäischen Bemühungen der Ukraine und die grundlegenden Fragen der gegenwärtigen Beziehungen zwischen beiden Ländern haben. In der Tat rief es keine politischen Spannungen hervor, dass das ukrainische Parlament genau am Tag des Besuchs von Staatspräsident Komorowski in Kiew ein Paket historischer Gesetze verabschiedete, das u. a. Angehörige der Organisation Ukrainischer Nationalisten, die im Zweiten Weltkrieg auch Verbrechen an Polen begangen hatte, als Freiheitskämpfer der Ukraine anerkannte.

Doch nicht weniger deutlich sind - sowohl in der politischen Elite als auch in der Bevölkerung - Ermüdungserscheinungen hinsichtlich des polnischen Engagements in dem Konflikt wie auch Befürchtungen, dass die Ukraine unabhängig vom bewaffneten Konflikt wieder einmal ihre Chance vertun könnte. Diese Stimmungsentwicklung äußert sich in verschiedenen Bereichen. Erstens ergibt sich das relativ bescheidene Engagement Warschaus für die finanzielle Unterstützung der Ukraine - ein Kredit in Höhe von 100 Mio. Euro für die Dauer von zehn Jahren ist nur ein Tropfen auf den heißen Stein - aus einer recht pessimistischen Bewertung des Reformprozesses des ukrainischen Staates und seiner Wirtschaft. Ein Jahr nach Beginn der Proteste auf dem Maidan lässt die Entschlossenheit, eine tiefgehende Umstrukturierung des Staates durchzuführen, viel zu wünschen übrig (und zwar nicht nur wegen des gleichzeitig geführten Krieges in der Ostukraine) und scheint die Stabilität des Regierungslagers nicht gegeben zu sein. Immer häufiger werden aus Warschau herbe Worte und Signale an die Ukraine gerichtet, dass deren Schicksal vor allem in den Händen der Ukrainer selbst liege.

Zweitens ist die öffentliche Meinung in Sachen Ukraine zurückhaltender, als dies gängige Meinungen über eine bedingungslose Unterstützung der Polen für die Ukraine suggerieren. Nach der oben angeführten Umfrage von CBOS vertreten 56 Prozent der Polen die Meinung, dass Polen die Ukraine nur gemeinsam mit anderen EU-Ländern unterstützen soll, 33 Prozent sind gegen ein Engagement Polens in diesem Konflikt. Nur sechs Prozent sind der Überzeugung, dass Warschau eine besondere Verantwortung trägt. 62 Prozent der Befragten wiederum sind der Ansicht, dass sich Polen nicht finanziell für die Ukraine engagieren sollte, gegenüber nur 31 Prozent, die dies befürworten. Sogar der von Ministerpräsidentin Kopacz versprochene Kredit von lediglich 100 Mio. Euro wird nur von 23 Prozent der Bevölkerung unterstützt.

Drittens ist es vor dem Hintergrund dieser Stimmungen wohl nicht verwunderlich, dass die Politiker versuchen, aus dieser eigentümlichen, wenngleich noch begrenzten Ukrainemüdigkeit vor den sich nähernden Präsidentschafts- und Parlamentswahlen Kapital zu schlagen. Kritische Stimmen zu einem - übermäßigen - Engagement Polens in Sachen Ukraine sind sogar aus den Reihen der Regierungskoalition zu vernehmen. Adam Jarubas, der Kandidat der Polnischen Bauernpartei (Polskie Stronnictwo Ludowe - PSL) für das Amt des 
Staatspräsidenten, kritisierte offen die Politik der Regierung und sprach sich für eine "gemäßigtere« Unterstützung Kiews aus. Der stellvertretende Ministerpräsident Janusz Piechociński, ebenfalls aus der PSL, warnte vor "Hunderttausenden" Migranten, die infolge der Krise nach Polen strömen könnten, und kritisierte die ukrainischen Eliten scharf für die Nichteinhaltung demokratischer und rechtsstaatlicher Standards. Die PSL repräsentiert die Interessen der Landwirte, die im Sommer 2014 unter den von Russland verhängten Gegensanktionen (vor allem Exportverbote für Fleisch und Ernährungs- und Agrarprodukte) litten. Nichtsdestotrotz, so hieß es in der Rzeczpospolita (5. März 2015), würde die Mehrheit der Branche gut damit zurechtkommen, sich auf andere Absatzmärkte umzustellen und könne der Export polnischer Produkte im Jahr 2015 sogar den Rekord von 21,4 Mrd. Euro übersteigen. Besonders offen ruft die Demokratische Linksallianz (Sojusz Lewicy Demokratycznej-SLD) dazu auf, die aktive Unterstützung der Ukraine zu beenden und Russland nicht zum Antagonisten zu machen. Deren Kandidatin, Małgorzata Ogórek, warf der Regierung sogar vor, »einen militärischen Konflikt mit Russland zu riskieren«, da sie die Möglichkeit der Waffenlieferung an die Ukraine nicht vollständig ausschlösse. Stimmen, dass in Polen eine "russische Partei« auftritt, im Sinne eines Milieus, das bereit zu Zugeständnissen gegenüber Moskau ist, mögen übertrieben sein, doch ist der gesellschaftliche und politische Konsens in Sachen Ostpolitik zweifellos schwächer als in der Vergangenheit.

\section{Sanktionen und Abschreckung}

Der geringe Einfluss Polens auf den diplomatischen Wettstreit um die Ukraine und der nüchterne Skeptizismus gegenüber den Fortschritten bei der Reformierung des Landes, der Zurückhaltung beim finanziellen Engagement zugunsten der Ukraine nahelegt, markieren die Grenzen der Möglichkeiten der polnischen Politik. Das Instrument, dessen Richtigkeit weiterhin keiner Diskussion unterliegt, bleiben die Wirtschaftssanktionen gegenüber Russland. Mitte Februar sagte Außenminister Schetyna in einem Fernsehinterview, dass "Sanktionen die einzige Waffe der EU« seien und verabschiedete sich damit noch einmal von eventuellen Plänen, der Ukraine Waffen zu liefern. Gleichzeitig unterstrich er damit die harte Haltung Polens in der Frage der Notwendigkeit, die ökonomischen Zwangsmaßnahmen gegenüber Russland als Antwort auf dessen Aktivitäten gegenüber der Ukraine aufrechtzuerhalten und gegebenenfalls zu verschärfen. Bereits Anfang Februar, nach der Offensive der Separatisten in der Ostukraine, war die polnische Regierung der Ansicht, dass es Zeit für eine weitere Runde von Sanktionen sei. Unter dem Einfluss Kiews, das die
Situation im Kontext der gerade stattfindenden Waffenstillstandsgespräche nicht verschärfen wollte, die schließlich zum Abkommen »Minsk II « führten, trat sie jedoch von dieser Forderung zurück. Allerdings sollte nach Meinung Warschaus die Androhung weiterer Sanktionen für den Fall aufrechterhalten werden, dass das Abkommen drastisch gebrochen würde. Die EU sollte bereit sein, groß angelegte Sanktionen in bestimmten Bereichen zu verhängen, falls die Russen die Demarkationslinie überschreiten und die territoriale Expansion fortsetzen würden. Ein eventueller Angriff auf Mariupol sei, so Schetyna, eine »rote Linie«, auf deren Überschreiten der Westen mit schrittweisen Sanktionen reagieren sollte, wobei der Ausschluss Russlands aus dem SWIFT-System der letzte Schritt sein sollte, vergleichbar mit einer ökonomischen »Nuklearoption«. Die aktuell geltenden Sanktionen sollten bis zur vollständigen Implementierung des Minsker Abkommens aufrechterhalten werden.

Unabhängig vom Schicksal des Abkommens herrscht in Warschau die Überzeugung, dass es die strategischen Ziele Russlands nicht ändern werde, zu denen der Aufbau bzw. die Stärkung des eigenen Einflussbereichs, die Verdrängung der USA aus Europa und die Schwächung der Europäischen Union von außen gehören. Zur langfristigen Strategie, auf diese Situation zu reagieren, gehören die Unterstützung der Ukraine - mit den genannten, wesentlichen Beschränkungen -, Sanktionen gegenüber Russland und die Stärkung der NATO und der EU in Bereichen, die unter dem Aspekt der Abschreckung und der Widerstandsfähigkeit gegenüber russischen Aktivitäten, die gegen diese Strukturen gerichtet sind, von zentraler Bedeutung sind. Letzteres scheint nach polnischer Einschätzung heute das wichtigste zu sein und betrifft drei Bereiche.

Erstens: Eine Priorität ist die Stärkung des Verteidigungspotentials und der militärischen Fähigkeiten des Landes. Ein politisch wichtiges Symbol dieses Denkens war, dass Verteidigungsminister Tomasz Siemoniak in der neuen Regierung in den Rang des stellvertretenden Ministerpräsidenten erhoben wurde, sowie die Entscheidung über die Erhöhung der Militärausgaben von 1,95 Prozent des Bruttoinlandsprodukts auf 2 Prozent. Praktisch drücken sich diese Maßnahmen in der Beschleunigung strategischer Entscheidungen beim Kauf der Armeeausstattung aus, insbesondere von Raketenabwehrsystemen und Kampfhubschraubern. Die Bekanntmachung der Einkäufe ist für den 21. April vorgesehen. Die Wahl der Anbieter (im Fall des größten Vertrags, der die Raketenabwehr betrifft und $20 \mathrm{Mrd}$. Zloty umfasst, wird sie zwischen dem US-amerikanischen Hersteller Raytheon und dem europäischen Hersteller Eurosam getroffen) wird weitreichende politische Folgen auch für die polnische Rüstungsindustrie haben. 
Zweitens misst Polen der Umsetzung der Beschlüsse des NATO-Gipfels in Newport große Bedeutung bei, insbesondere was die Stärkung der Ostflanke des Bündnisses betrifft. Die Bildung der sogenannten Speerspitze, deren Hauptquartier sich in Stettin befinden soll, rotierende Manöver der NATO-Truppen auf polnischem Territorium und in anderen Ländern Ostmitteleuropas sowie der Readiness Action Plan, der detaillierte Vorgaben für das Bündnis im Falle einer Bedrohung enthält, sind die wichtigsten Aspekte des dort beschlossenen Pakets. Dennoch wird ihre Bedeutung letztlich davon abhängen, wie die Beschlüsse in der Praxis interpretiert und angewendet werden. Polen legt Wert darauf, dass sich die kollektive Verteidigung der NATO auf »konventionelle Bedrohungen der Sicherheit" ausrichten solle, wie sie in Artikel 5 des Nordatlantikpakts vorgesehen werden. Anders gesagt - die neuen Instrumente der NATO sollten so konstruiert sein, dass sie in erster Linie auf die Herausforderungen antworten, mit denen sich das Bündnis an der Ostflanke messen muss. Warschau unterstreicht, dass die Einführung dieser Instrumente eine Antwort auf die Krise im Osten war - ohne die Annexion der Krim wären es weder zum Readiness Action Plan noch zur "Speerspitze« gekommen. Allerdings trifft diese Haltung unvermeidlich auf den Widerstand der südlichen Bündnispartner, die über weniger konventionelle Bedrohungen, wie sie aus dem Chaos im Nahen Osten und in Nordafrika resultieren, besorgt sind.

Drittens gehört Polen zu denjenigen Ländern, die die Energieunion am entschiedensten unterstützen. Diese soll nicht nur dem Aufbau eines gemeinsamen Energiemarktes dienen, sondern auch die EU in Verhandlungen mit externen Partnern wie zum Beispiel Gazprom deutlich stärken. Die Energieunion wird als Schlüssel- projekt für die Stärkung der Einheit der EU betrachtet sowie für ihre Fähigkeit, sich gegen Praktiken zu stellen, die gegen die Interessen einzelner Mitgliedsländer gerichtet sind. Zwar wurde der polnischen Forderung gemeinsamer Energiekäufe in der EU, die im Frühjahr 2014 noch von Ministerpräsident Donald Tusk publik gemacht wurde, nicht stattgegeben, aber das Kommuniqué der Europäischen Kommission zur Energieunion vom März 2015 erfüllte den Großteil der polnischen Erwartungen. Polen wird sich mit Sicherheit weiter vehement nicht nur für den Ausbau der Verbindungsleitungen in Europa aussprechen, sondern auch für den Beschluss von Grundsätzen zur Transparenz von Energieverträgen einzelner EU-Mitgliedsländer sowie für die größtmögliche Beteiligung der Europäischen Kommission bei den Verhandlungen.

Die Präsidentschaftswahlen im Mai, deren Sieger mit dem amtierenden Bronisław Komorowski bereits festzustehen scheint (offen bleibt nur, ob ein oder zwei Wahlgänge nötig sein werden), beeinflussen die polnische Außenpolitik nicht wesentlich. Das Ergebnis des Kandidaten der Opposition, Andrzej Duda, wird allerdings als Maß für das Mobilisierungspotential von Recht und Gerechtigkeit (Prawo i Sprawiedliwość - PiS) für die im Herbst stattfinden Parlamentswahlen gelten. Im Wahljahr 2015 sind keine ambitionierteren oder entschiedeneren Aktivitäten auf der internationalen Bühne zu erwarten - Umfragen zeigen, dass die Ukraine-Politik nicht unbedingt das Thema ist, mit dem sich Wählerstimmen gewinnen lassen. Auch nach den Wahlen werden weder die Prioritäten noch die genannten Einschränkungen der polnischen Politik gegenüber dem Konflikt im Osten eine grundlegende Änderung erfahren.

Übersetzung aus dem Polnischen: Silke Plate

Über den Autor

Piotr Buras, Politologe, Journalist, Deutschland- und Europaexperte, ist seit 2013 Leiter des Warschauer Büros des European Council on Foreign Relations - ECFR. Vorher war er fünf Jahre als Journalist der Gazeta Wyborcza in Berlin tätig. 


\section{Umfragen zum Ukraine-Konflikt und zum Verhältnis gegenüber anderen Nationen}

Grafik 1: Interessieren Sie sich für die Ereignisse in der Ukraine oder nicht? (\%)

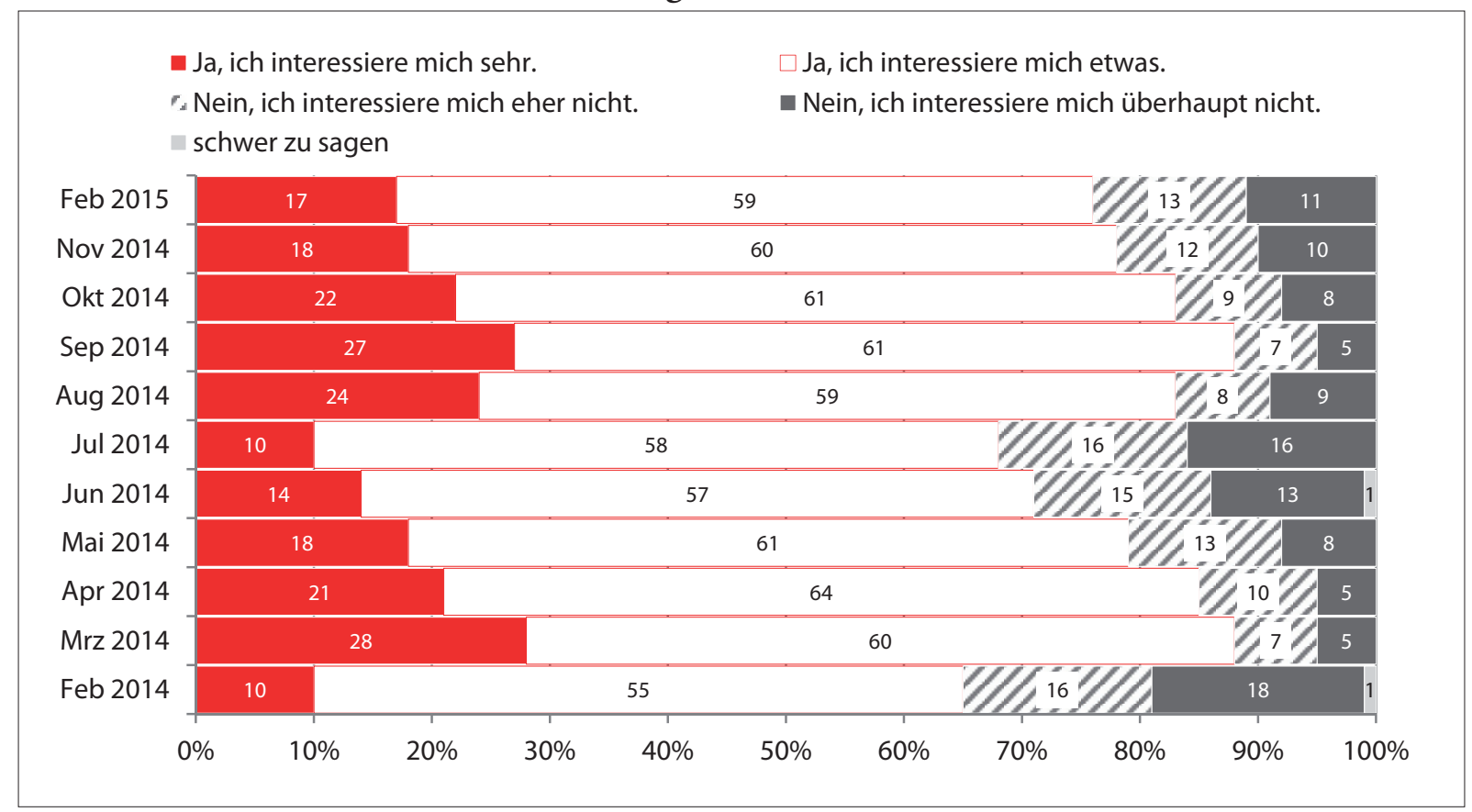

Quelle: CBOS Nr 22/2015: O sytuacji na Ukrainie i polskiej pomocy dla wschodniego sasiada [Über die Situation in der Ukraine und die polnische Hilfe für den östlichen Nachbarn]. Warszawa 02/2015. <www.cbos.pl>

Grafik 2: Bedroht Ihrer Meinung nach die Situation in der Ukraine die Sicherheit Polens oder nicht? (\%)

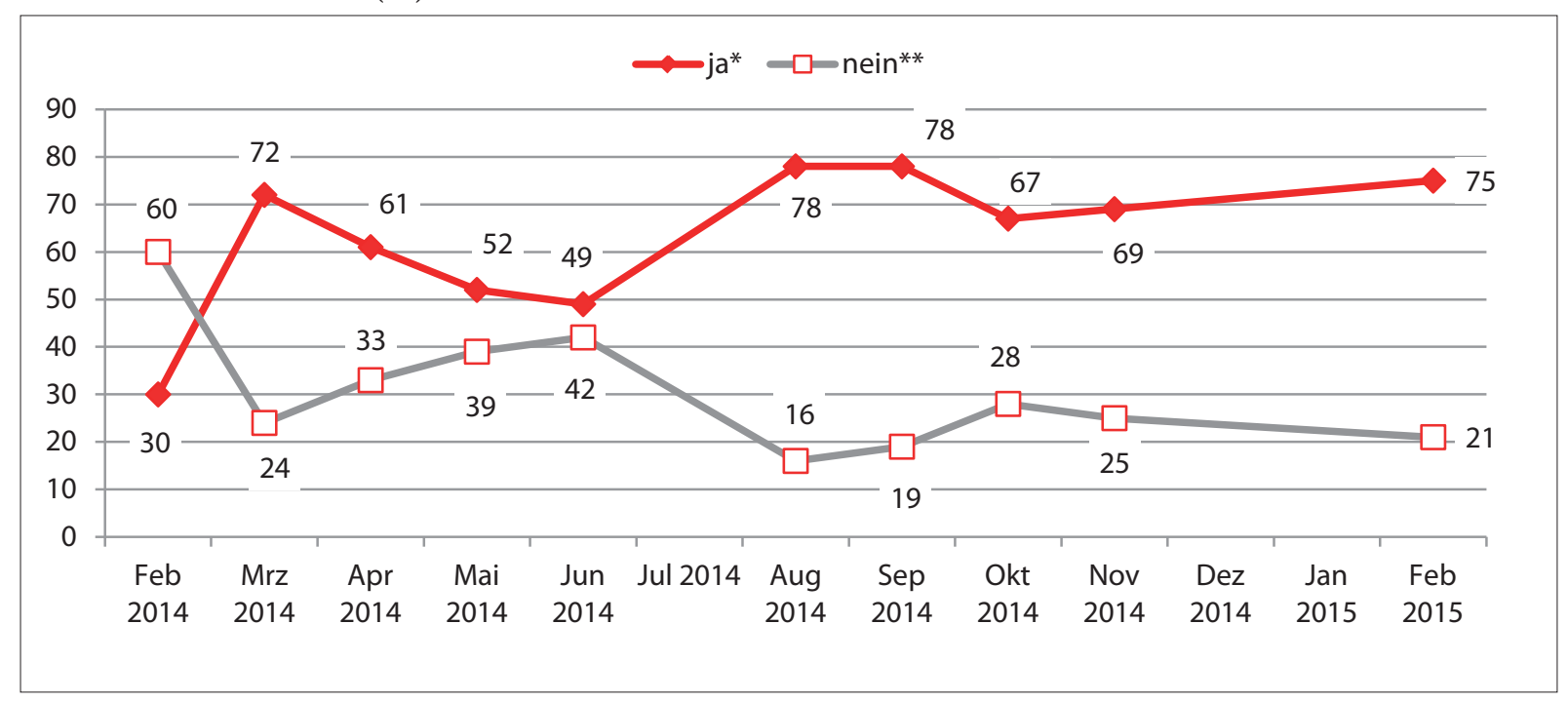

*Die Antworten "eindeutig ja" und "eher ja" wurden zusammengefasst.

** Die Antworten "eindeutig nein" und "eher nein " wurden zusammengefasst.

Die Antwort "schwer zu sagen" wird nicht aufgeführt.

Quelle: CBOS Nr 22/2015: O sytuacji na Ukrainie i polskiej pomocy dla wschodniego sąsiada [Über die Situation in der Ukraine und die polnische Hilfe für den östlichen Nachbarn]. Warszawa 02/2015. <www.cbos.pl> 
Grafik 3: Bedroht Ihrer Meinung nach die Situation in der Ukraine die Sicherheit Europas oder nicht? (\%)

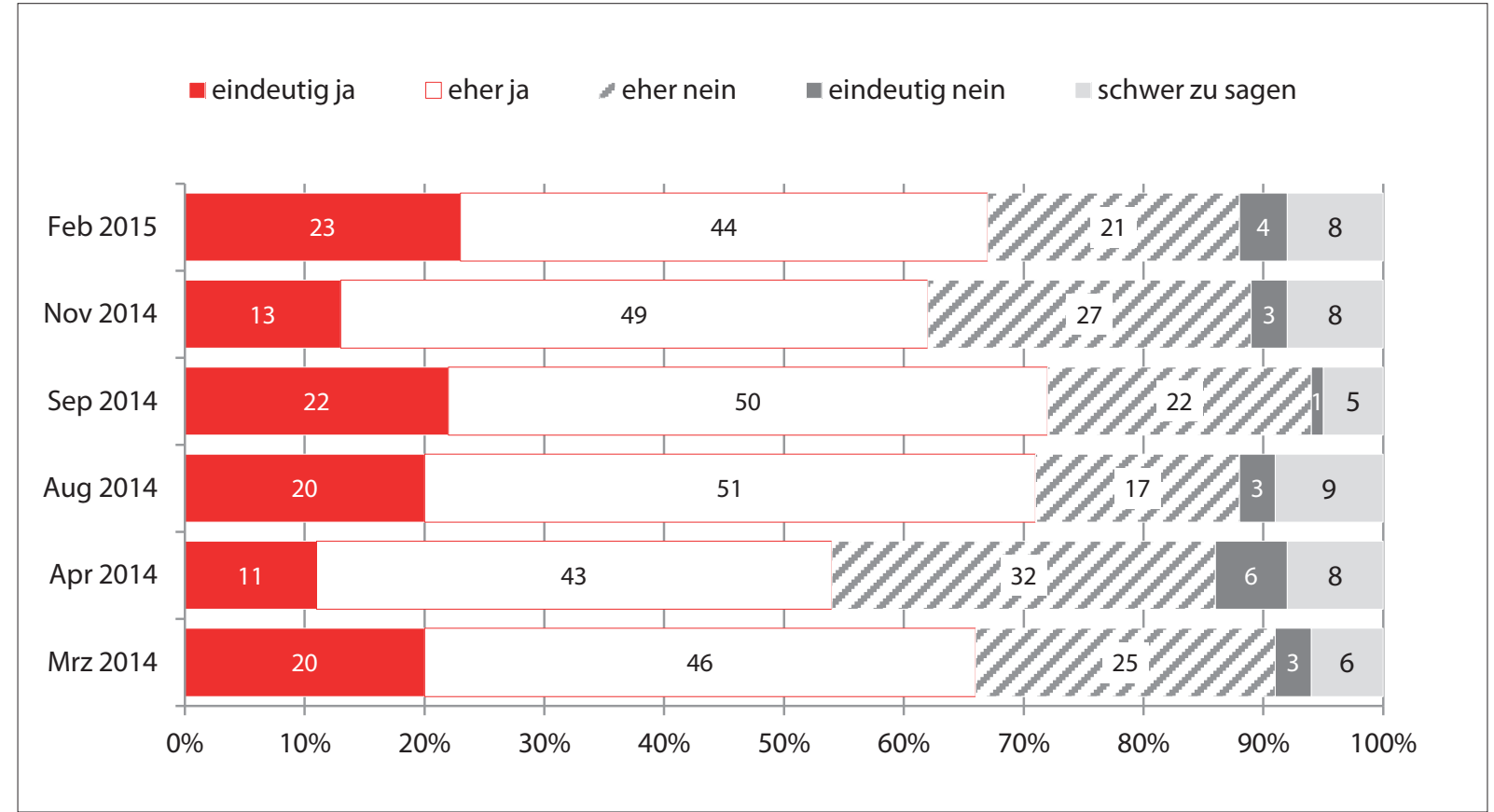

Quelle: CBOS Nr 22/2015: O sytuacji na Ukrainie i polskiej pomocy dla wschodniego sasiada [Über die Situation in der Ukraine und die polnische Hilfe für den östlichen Nachbarn]. Warszawa 02/2015. <www.cbos.pl>

Grafik 4: Bedroht Ihrer Meinung nach die Situation in der Ukraine die Sicherheit in der Welt oder nicht? (\%)

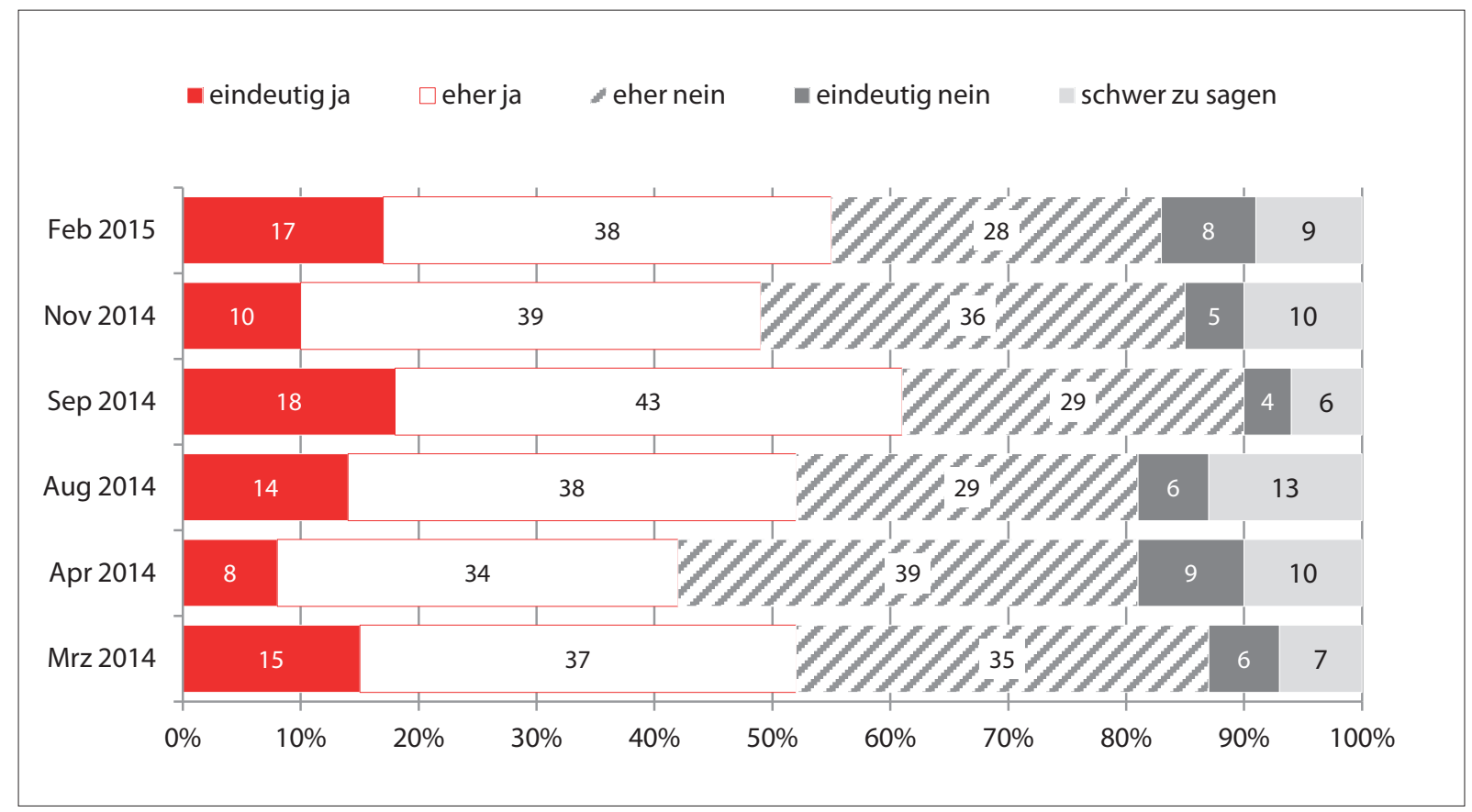

Quelle: CBOS Nr 22/2015: O sytuacji na Ukrainie i polskiej pomocy dla wschodniego sąsiada [Über die Situation in der Ukraine und die polnische Hilfe für den östlichen Nachbarn]. Warszawa 02/2015. <www.cbos.pl> 
Grafik 5: Wie sollte sich Polen Ihrer Meinung nach angesichts der Ereignisse in der Ukraine verhalten? Welche Meinung ist Ihnen am nächsten? (\%)

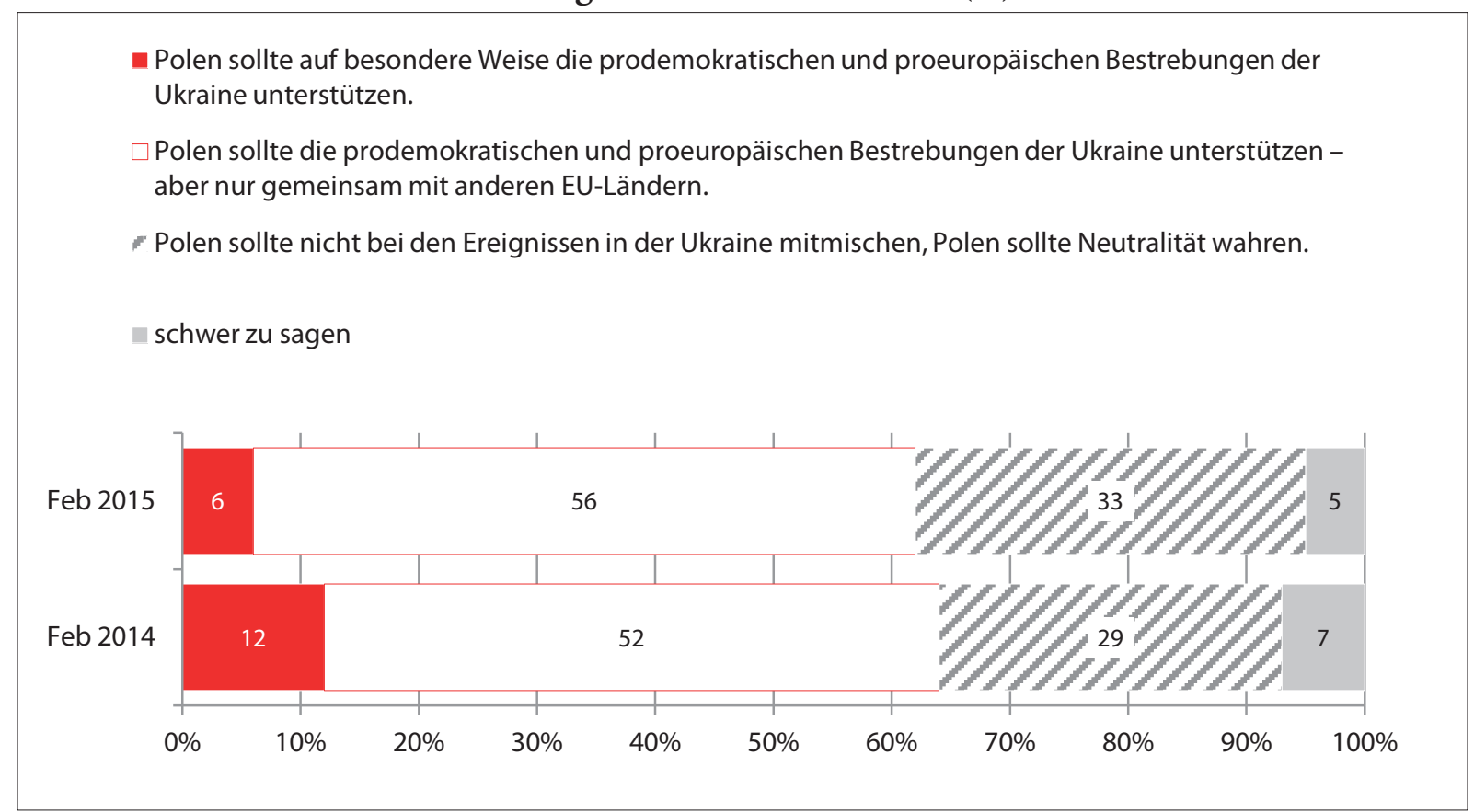

Quelle: CBOS Nr 22/2015: O sytuacji na Ukrainie i polskiej pomocy dla wschodniego sasiada [Über die Situation in der Ukraine und die polnische Hilfe für den östlichen Nachbarn]. Warszawa 02/2015. <www.cbos.pl>

Grafik 6: Sollte Polen Ihrer Meinung nach die Ukraine finanziell unterstützen?

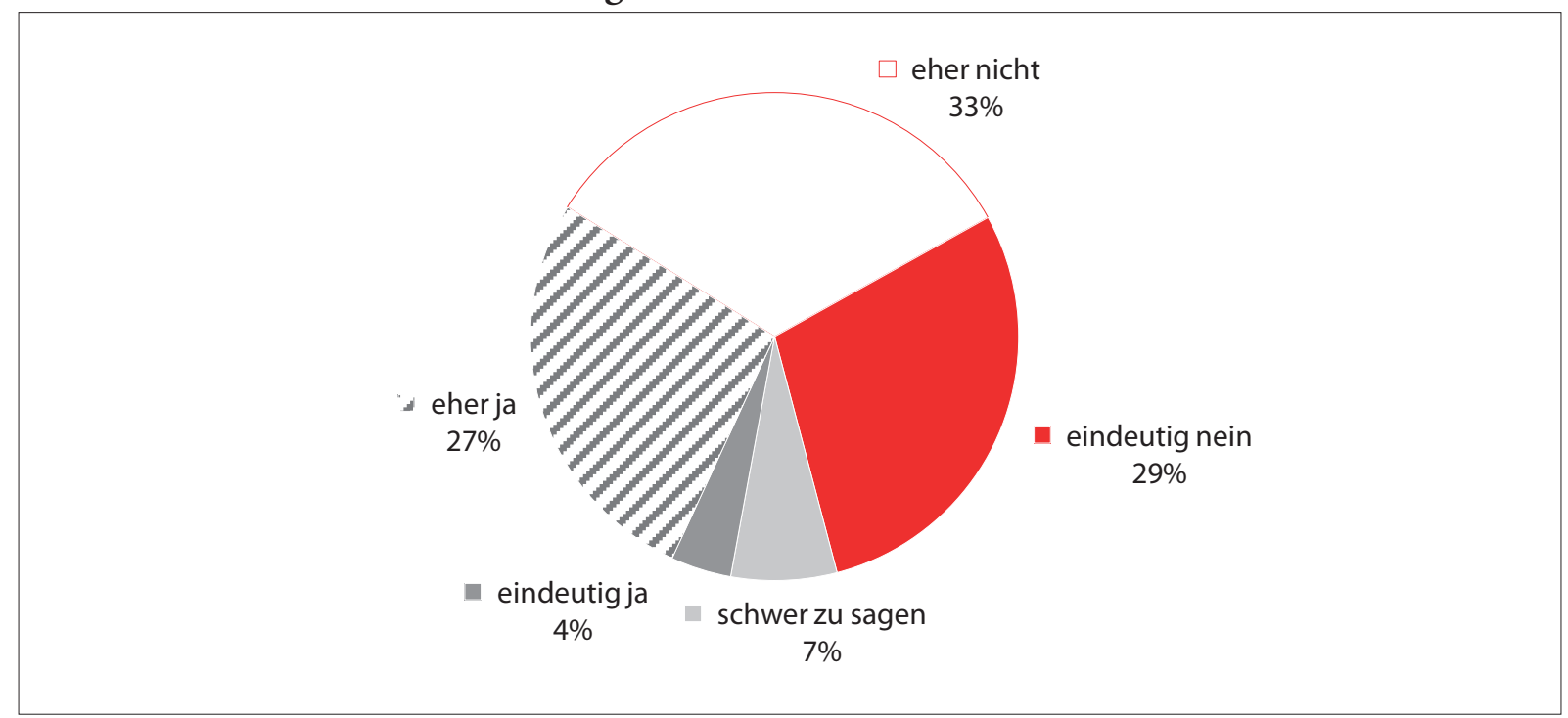

Quelle: CBOS Nr 22/2015: O sytuacji na Ukrainie i polskiej pomocy dla wschodniego sąsiada [Über die Situation in der Ukraine und die polnische Hilfe für den östlichen Nachbarn]. Warszawa 02/2015. <www.cbos.pl> 
Grafik 7: Kürzlich hat Ministerpräsidentin Ewa Kopacz bei Ihrem Besuch in Kiew der Ukraine einen Kredit in Höhe von 100 Mio. Euro zur Unterstützung der ukrainischen Wirtschaft angeboten. Ist die angebotene Hilfe Ihrer Meinung nach...

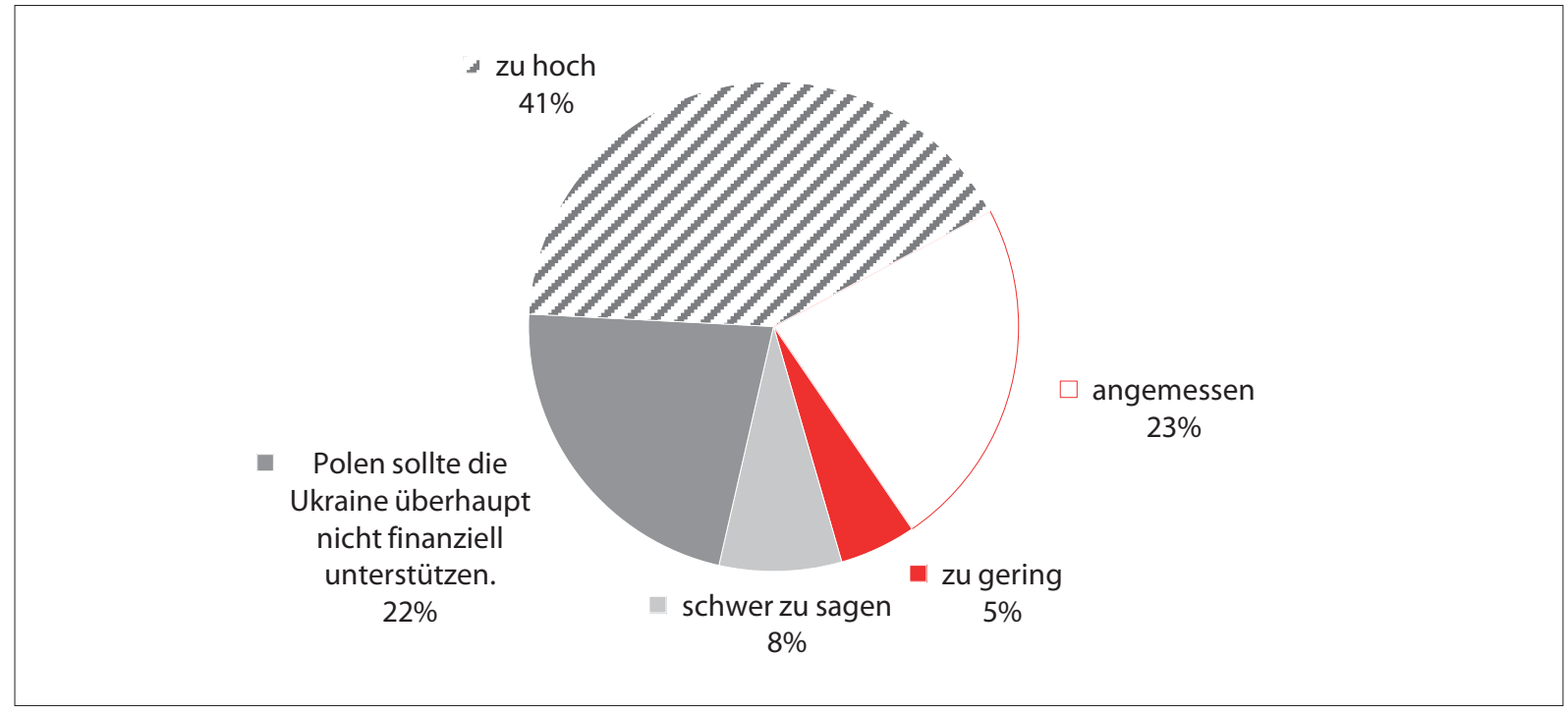

Quelle: CBOS Nr 22/2015: O sytuacji na Ukrainie i polskiej pomocy dla wschodniego sasiada [Über die Situation in der Ukraine und die polnische Hilfe für den östlichen Nachbarn]. Warszawa 02/2015. <www.cbos.pl>

Grafik 8: Die Europäische Union hat als Antwort auf die Annexion der Krim und den Einsatz Russlands im Konflikt in der Ostukraine Wirtschaftssanktionen gegenüber Russland verhängt. Sind die Wirtschaftssanktionen gegenüber Russland Ihrer Meinung nach... $(\%)$

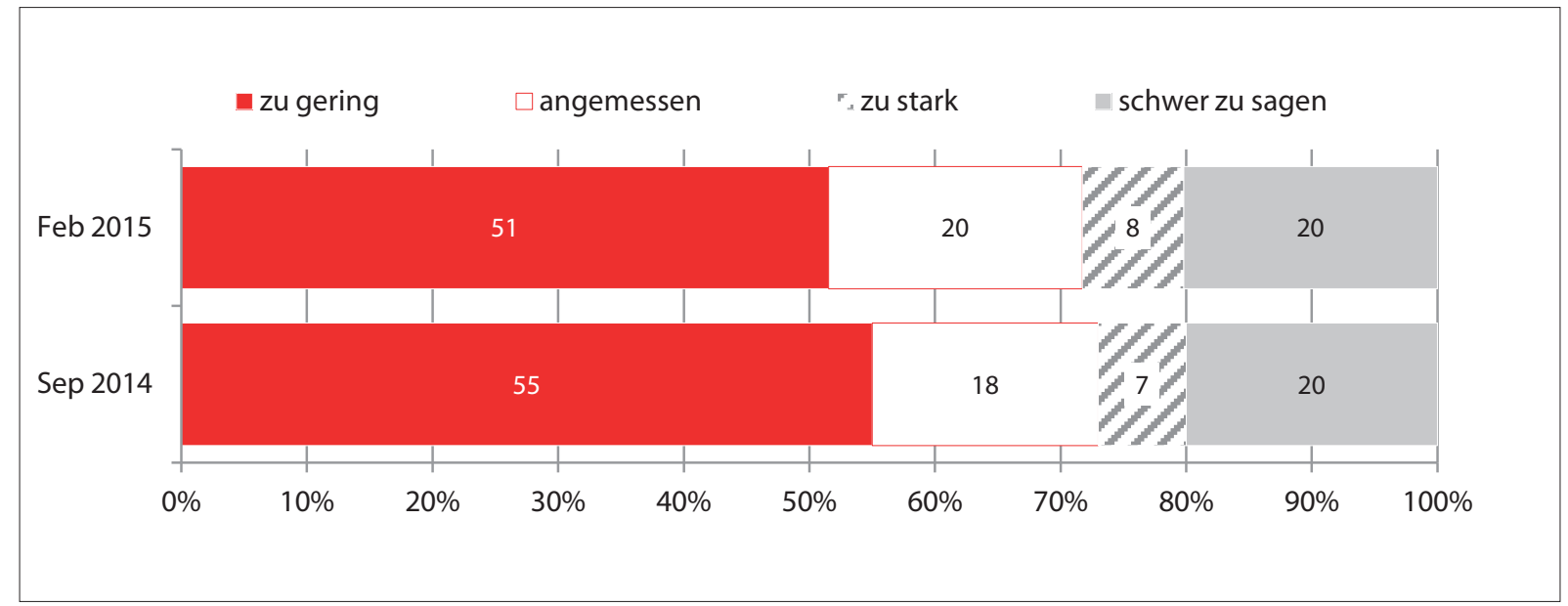

Quelle: CBOS Nr 22/2015: O sytuacji na Ukrainie i polskiej pomocy dla wschodniego sasiada [Über die Situation in der Ukraine und die polnische Hilfe für den östlichen Nachbarn]. Warszawa 02/2015. <www.cbos.pl> 
Tabelle 1: Welches der Ereignisse im Jahr 2014 war Ihrer Meinung nach das wichtigste für Polen?

\begin{tabular}{|c|c|}
\hline die Wahl Donald Tusks zum Präsidenten des Europäischen Rates & $17 \%$ \\
\hline die Wahlen auf der Ebene der Selbstverwaltung & $9 \%$ \\
\hline $\begin{array}{l}\text { der ukrainisch-russische Konflikt, die Annexion der Krim, die Situation in der Ukraine und } \\
\text { deren politisches und wirtschaftliches Echo }\end{array}$ & $7 \%$ \\
\hline $\begin{array}{l}\text { die Volleyball-Weltmeisterschaft der Männer in Polen, der sportliche und organisatorische } \\
\text { Erfolg Polens }\end{array}$ & $6 \%$ \\
\hline der Wechsel des Ministerpräsidenten und der Regierung & $4 \%$ \\
\hline die Heiligsprechung Johannes Pauls II. & $3 \%$ \\
\hline andere sportliche Erfolge und Ereignisse & $2 \%$ \\
\hline die Entwicklung der Straßen- und Gleisinfrastruktur & $1 \%$ \\
\hline die »Abhöraffäre» & $1 \%$ \\
\hline die Unterzeichnung des Klimapakets durch Polen & $1 \%$ \\
\hline $\begin{array}{l}\text { die Beendigung des Afghanistan-Einsatzes polnischer Soldaten im Rahmen der bisherigen } \\
\text { NATO-Mission }\end{array}$ & $1 \%$ \\
\hline $\begin{array}{l}\text { andere politische Ereignisse (u. a. die Feierlichkeiten zum 25-jährigen Jubiläum der Demo- } \\
\text { kratie in Polen, der "Marsch der Unabhängigkeit«, die Verbrennung des Regenbogens [eines } \\
\text { Kunstwerks in Warschau]) }\end{array}$ & $1 \%$ \\
\hline $\begin{array}{l}\text { andere Ereignisse (u. a. Zuwendungen der EU; die Verabschiedung des EU-Budgets für die } \\
\text { Jahre } 2014 \text { bis 2020; die Charta der Großfamilien; wissenschaftliche und medizinische Erfolge) }\end{array}$ & $2 \%$ \\
\hline Schwer zu sagen; ich weiß nicht; mir fällt nichts ein. & $39 \%$ \\
\hline Es gab kein solches Ereignis; es hat sich nichts Besonderes ereignet. & $10 \%$ \\
\hline
\end{tabular}

Anm.: Die Gesamtsumme ergibt mehr als $100 \%$, da die Befragten mehrere Ereignisse nennen konnten.

Quelle: CBOS Nr 4/2015: Wydarzenie roku 2014 w Polsce i na świecie [Das Ereignis des Jahres 2014 in Polen und in der Welt]. Warszawa 01/2015. <www.cbos.pl>

\section{Tabelle 2: Welches der Ereignisse des Jahres 2014 kann man, Ihrer Meinung nach, als das wichtigste für die Welt bestimmen?}

\begin{tabular}{|c|c|}
\hline $\begin{array}{l}\text { der ukrainisch-russische Konflikt, die Annexion der Krim, der Krieg in der Ukraine, die } \\
\text { aggressive Politik des Präsidenten Wladimir Putin }\end{array}$ & $36 \%$ \\
\hline $\begin{array}{l}\text { die europäische und internationale Solidarität als Antwort auf die Politik Russlands, die } \\
\text { Wirtschaftssanktionen, die Haltung der USA u. ä. }\end{array}$ & $3 \%$ \\
\hline $\begin{array}{l}\text { die Situation im Nahen Osten, die Entstehung des Islamischen Staates und die von ihm } \\
\text { ausgehende Bedrohung }\end{array}$ & $2 \%$ \\
\hline $\begin{array}{l}\text { die Eskalation von Konflikten im Allgemeinen und der konstatierte Anstieg der Bedrohung } \\
\text { des Friedens in Europa und in der Welt }\end{array}$ & $1 \%$ \\
\hline der Kiewer Maidan & $1 \%$ \\
\hline der Abschuss des malaysischen Flugzeugs über dem Territorium der Ukraine & $1 \%$ \\
\hline die Ebolaepidemie & $1 \%$ \\
\hline die Heiligsprechung Johannes Pauls II. & $1 \%$ \\
\hline das Wirken Papst Franziskus' & $1 \%$ \\
\hline die Wahl Donald Tusks zum Präsidenten des Europäischen Rates & $1 \%$ \\
\hline der Preisverfall für Erdöl & $1 \%$ \\
\hline $\begin{array}{l}\text { sportliche Ereignisse: die in Polen ausgetragene Volleyball-Weltmeisterschaft der Männer und } \\
\text { die Fußballweltmeisterschaft in Brasilien }\end{array}$ & $1 \%$ \\
\hline wissenschaftliche Entdeckungen und Erfolge & $1 \%$ \\
\hline Maßnahmen zur Friedenssicherung in der Welt, allgemein & $1 \%$ \\
\hline andere Ereignisse & $2 \%$ \\
\hline Schwer zu sagen; ich weiß nicht. & $46 \%$ \\
\hline Es gab kein solches Ereignis. & $6 \%$ \\
\hline
\end{tabular}


Grafik 9: Wie bewerten Sie die gegenwärtigen Beziehungen zwischen Polen und seinen nächsten Nachbarn? (\%)

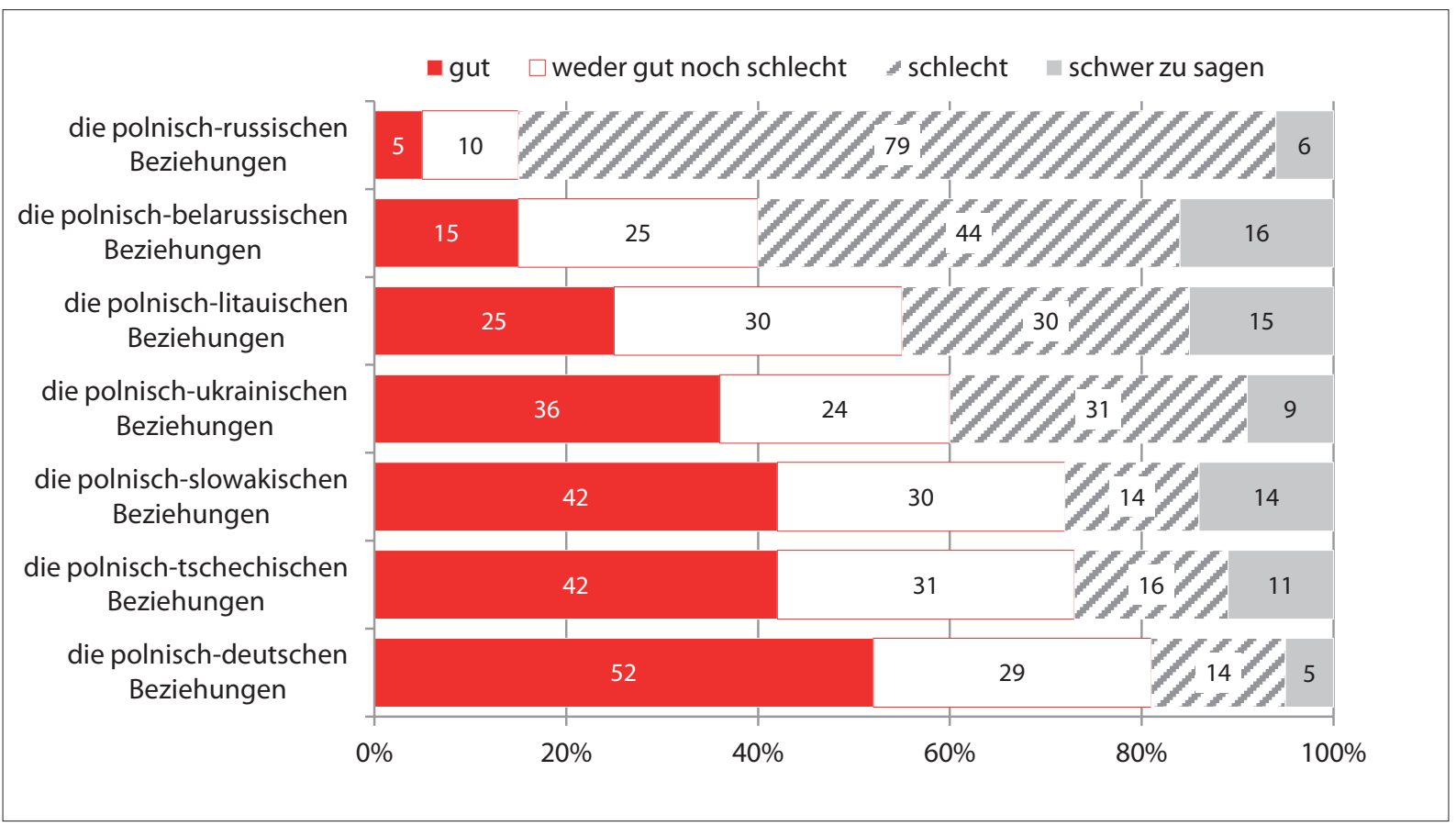

Quelle: CBOS Nr 30/2015: O stosunkach z sasiednimi krajami - relacje polityczne a nastawienie do nacji [Über die Beziehungen zu den Nachbarländern - die politischen Beziehungen und die Einstellung zur Nation]. Warszawa 03/2015. <www cbos.pl>

Grafik 10: Sympathie und Antipathie gegenüber Russen und Ukrainern (\%)

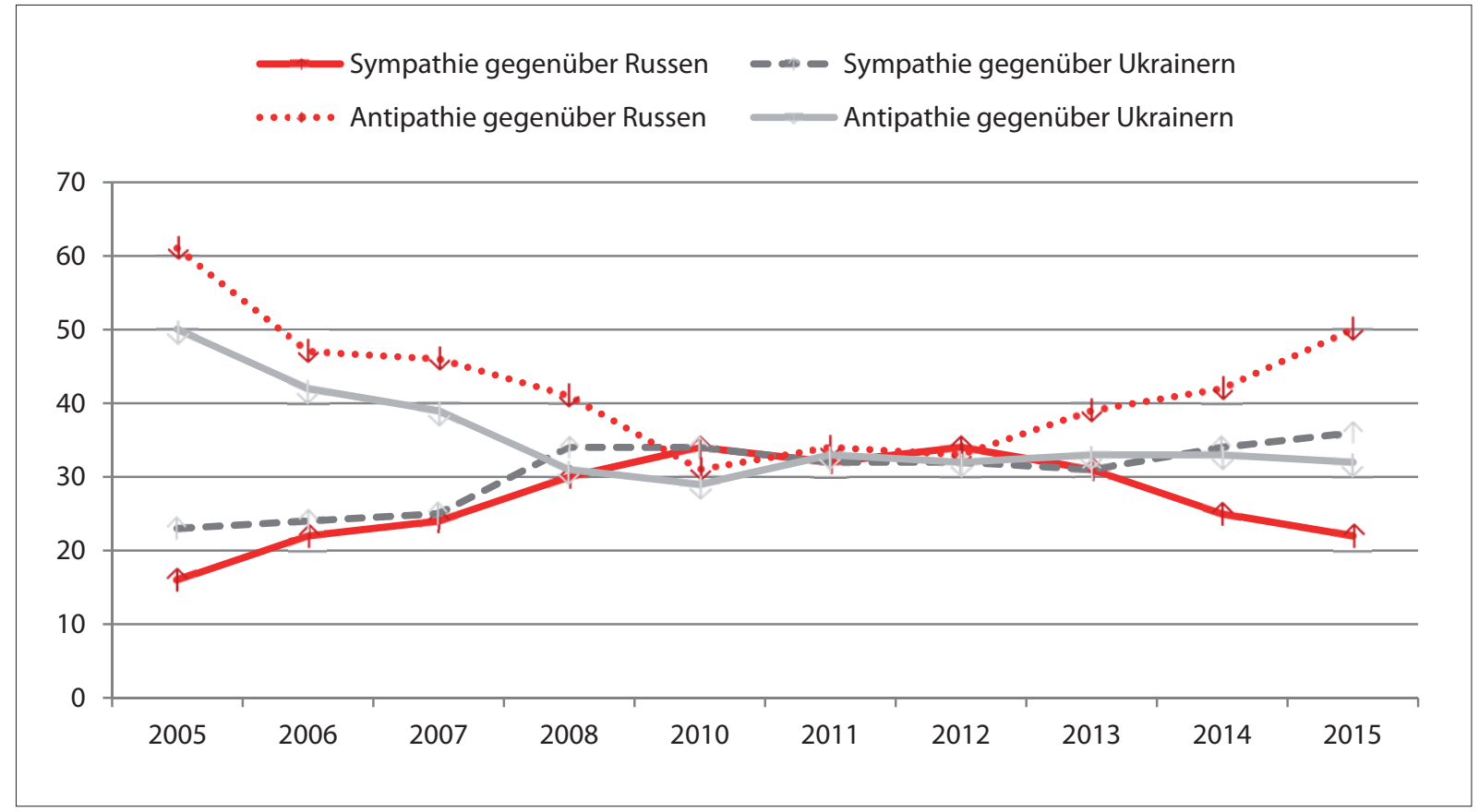

Anm.: Siehe Tabelle 3 und 4 auf der nächsten Seite für die genauen Werte.

Quelle: CBOS Nr 14/2015: Stosunek do innych narodów [Das Verhältnis zu anderen Nationen]. Warszawa 01/2015. <www.cbos.pl> 
Tabelle 3: Sympathie gegenüber einzelnen Nationen (\%)

\begin{tabular}{|l|c|c|c|c|c|c|c|c|c|c|}
\hline & 2005 & 2006 & 2007 & 2008 & 2010 & 2011 & 2012 & 2013 & 2014 & 2015 \\
\hline Belarussen & 18 & 23 & 27 & 34 & 34 & 31 & 33 & 30 & 29 & 28 \\
\hline Briten & 50 & 50 & 53 & 51 & 50 & 44 & 54 & 47 & 43 & 48 \\
\hline Bulgaren & 19 & 24 & 29 & 35 & 37 & 33 & 38 & 32 & 31 & 32 \\
\hline Deutsche & 34 & 33 & 30 & 38 & 39 & 38 & 43 & 38 & 39 & 43 \\
Esten & - & - & - & - & - & - & - & - & 30 & 29 \\
\hline Franzosen & 43 & 48 & 52 & 49 & 51 & 45 & 53 & 41 & 44 & 44 \\
Georgier & - & - & 27 & 35 & 32 & 34 & 37 & 31 & 31 & 32 \\
Griechen & 41 & 47 & 49 & 46 & 47 & 43 & 40 & 35 & 37 & 39 \\
Italiener & 52 & 52 & 51 & 54 & 52 & 47 & 55 & 46 & 48 & 51 \\
Litauer & 32 & 36 & 38 & 41 & 42 & 36 & 40 & 32 & 34 & 34 \\
Russen & 16 & 22 & 24 & 30 & 34 & 32 & 34 & 31 & 25 & 22 \\
\hline Rumänen & 12 & 16 & 16 & 25 & 26 & 24 & 26 & 21 & 21 & 21 \\
\hline Slowaken & 36 & 44 & 48 & 51 & 51 & 49 & 57 & 48 & 47 & 48 \\
\hline Spanier & 47 & 53 & 49 & 52 & 51 & 47 & 53 & 45 & 46 & 49 \\
\hline Tschechen & 46 & 52 & 53 & 53 & 53 & 51 & 58 & 51 & 50 & 50 \\
\hline Ukrainer & 23 & 24 & 25 & 34 & 34 & 32 & 32 & 31 & 34 & 36 \\
\hline Ungarn & 39 & 45 & 45 & 48 & 49 & 46 & 52 & 42 & 43 & 44 \\
\hline US-Ame- & 46 & 49 & 44 & 47 & 45 & 43 & 51 & 43 & 41 & 44 \\
rikaner & & & & & & & & & & \\
\hline
\end{tabular}

Quelle: CBOS Nr 14/2015: Stosunek do innych narodów [Das Verhältnis zu anderen Nationen]. Warszawa 01/2015. <www.cbos.pl>

Tabelle 4: Antipathie gegenüber einzelnen Nationen (\%)

\begin{tabular}{|l|c|c|c|c|c|c|c|c|c|c|}
\hline & 2005 & 2006 & 2007 & 2008 & 2010 & 2011 & 2012 & 2013 & 2014 & 2015 \\
\hline Belarussen & 51 & 39 & 34 & 26 & 23 & 26 & 26 & 31 & 31 & 29 \\
\hline Briten & 19 & 15 & 14 & 15 & 12 & 16 & 15 & 15 & 20 & 15 \\
\hline Bulgaren & 42 & 34 & 28 & 20 & 18 & 20 & 20 & 25 & 28 & 25 \\
\hline Deutsche & 38 & 33 & 39 & 32 & 28 & 27 & 24 & 28 & 30 & 22 \\
\hline Esten & - & - & - & - & - & - & - & - & 22 & 21 \\
\hline Franzosen & 25 & 16 & 14 & 15 & 10 & 14 & 13 & 17 & 18 & 16 \\
Georgier & - & - & 28 & 25 & 22 & 18 & 21 & 22 & 25 & 25 \\
\hline Griechen & 21 & 17 & 12 & 14 & 11 & 14 & 21 & 25 & 23 & 18 \\
\hline Italiener & 15 & 14 & 13 & 12 & 10 & 13 & 11 & 15 & 15 & 11 \\
\hline Litauer & 31 & 24 & 22 & 17 & 15 & 19 & 23 & 25 & 27 & 22 \\
\hline Russen & 61 & 47 & 46 & 41 & 31 & 34 & 33 & 39 & 42 & 50 \\
\hline Rumänen & 62 & 52 & 51 & 40 & 35 & 40 & 39 & 41 & 45 & 43 \\
\hline Slowaken & 26 & 18 & 15 & 13 & 11 & 11 & 10 & 13 & 15 & 12 \\
\hline Spanier & 18 & 12 & 13 & 10 & 8 & 9 & 10 & 15 & 14 & 12 \\
\hline Tschechen & 22 & 14 & 15 & 12 & 9 & 12 & 11 & 13 & 17 & 14 \\
\hline Ukrainer & 50 & 42 & 39 & 31 & 29 & 33 & 32 & 33 & 33 & 32 \\
\hline Ungarn & 25 & 18 & 16 & 13 & 10 & 12 & 13 & 16 & 17 & 14 \\
\hline $\begin{array}{l}\text { US-Ame- } \\
\text { rikaner }\end{array}$ & 24 & 16 & 21 & 18 & 16 & 19 & 16 & 21 & 21 & 17 \\
\hline
\end{tabular}

Quelle: CBOS Nr 14/2015: Stosunek do innych narodów [Das Verhältnis zu anderen Nationen]. Warszawa 01/2015. <www.cbos.pl> 


\section{März 2015 - 20. April 2015}

\begin{tabular}{|c|c|}
\hline 17.03 .2015 & $\begin{array}{l}\text { Die Regierung beschließt für die Jahre } 2015 \text { bis } 2020 \text { das Programm "Senior-WIGOR«, das mit medizini- } \\
\text { scher Hilfe, Rehabilitationsmaßnahmen, Sozialberatungen und Freizeitmöglichkeiten auf die größer werdende } \\
\text { Gruppe der Senioren in der Bevölkerung ausgerichtet ist. Insgesamt sollen } 370 \text { Mio. Zloty bereitgestellt werden. }\end{array}$ \\
\hline 18.03 .2015 & $\begin{array}{l}\text { Außenminister Grzegorz Schetyna bezeichnet die Äußerung des russischen Präsidenten Wladimir Putin, dass } \\
\text { die Demonstranten auf dem Kiewer Maidan (Ukraine) in Polen geschult worden seien, als absurd. Polen werde } \\
\text { damit als Land dargestellt, das sich Russland gegenüber niederträchtig und aggressiv verhalte und einen hybri- } \\
\text { den Krieg gegen Russland führe. Diese Lüge diene der Propaganda im eigenen Land, die Russland als bedroh- } \\
\text { tes Land darstelle, das um seine Zukunft und seine Werte kämpfen müsse. }\end{array}$ \\
\hline 19.03 .2015 & $\begin{array}{l}\text { Staatspräsident Bronisław Komorowski, der Chef des Büros für Nationale Sicherheit (Biuro Bezpieczeństwa Naro- } \\
\text { dowego - BBN), Stanisław Koziej, Innenministerin Teresa Piotrowska und Außenminister Grzegorz Schetyna } \\
\text { beraten über die Folgen des Anschlags am Vortag in Tunis, bei dem polnische Staatsbürger verletzt wurden und } \\
\text { ums Leben kamen, über die Sicherheit der diplomatischen Vertretungen Polens und über Antiterrormaßnahmen. }\end{array}$ \\
\hline 20.03 .2015 & $\begin{array}{l}\text { Nach kontroverser Debatte verabschiedet der Sejm ein Gesetz zur finanziellen Unterstützung von Aktivisten } \\
\text { der antikommunistischen Opposition und politisch Verfolgten in der Volksrepublik Polen zwischen } 1956 \text { und } \\
\text { 1989. Die Unterstützung soll nur Personen in schwierigen materiellen Verhältnissen zukommen. }\end{array}$ \\
\hline 21.03 .2015 & $\begin{array}{l}\text { Verteidigungsminister Tomasz Siemoniak und der US-amerikanische Botschafter Stephen Mull besuchen in } \\
\text { Sochaczew (Woiwodschaft Masowien) eine polnisch-US-amerikanische Militärübung im Rahmen der NATO- } \\
\text { Übung "Atlantic Resolve«. Der Ort der Übung in der Nähe Warschaus sei eine Antwort auf die Ereignisse im } \\
\text { östlichen Europa. Siemoniak hebt hervor, dass Polen in der Vergangenheit schlechte Erfahrungen mit der Anwe- } \\
\text { senheit ausländischen Militärs gemacht habe, jedoch die US-amerikanische Armee sowohl von Seiten der Poli- } \\
\text { tik als auch der Bevölkerung willkommen sei. }\end{array}$ \\
\hline 22.03 .2015 & $\begin{array}{l}\text { In seiner Rede auf dem Brussels Forum, das vom German Marshall Fund veranstaltet wird, stellt Staatspräsi- } \\
\text { dent Bronisław Komorowski fest, dass die Zeit der Friedensdividende nach dem Ende des Kalten Krieges been- } \\
\text { det sei. Ereignisse wie die Annexion der ukrainischen Halbinsel Krim durch Russland würden ein grundlegen- } \\
\text { des Umdenken in der Politik des Westens gegenüber Russland erfordern. Der Westen habe sich zu lange der } \\
\text { Illusion hingegeben, dass Russland den Weg der Modernisierung und Demokratisierung einschlägt. Notwen- } \\
\text { dig sei eine Strategie, die den Westen angesichts der neuen Herausforderungen vereine. In diesem Zusammen- } \\
\text { hang spricht sich Komorowski für einen "Marshallplan« für die Ukraine und für das Freihandelsabkommen } \\
\text { TTIP zwischen der EU und den USA aus. }\end{array}$ \\
\hline 23.03 .2015 & $\begin{array}{l}\text { Eine Delegation des Vorstands der polnisch-deutschen Parlamentariergruppe von Sejm und Senat beginnt unter } \\
\text { der Leitung ihres stellvertretenden Vorsitzenden, Senator Aleksander Swwieykowski, ihren viertägigen Besuch } \\
\text { in Deutschland. In Frankfurt (Oder) und Słubice informiert sie sich über die Zusammenarbeit und gemein- } \\
\text { samen Projekte an der Grenze und führt in Berlin politische Gespräche im Bundestag und mit Vertretern der } \\
\text { Bundesregierung. }\end{array}$ \\
\hline 24.03 .2015 & $\begin{array}{l}\text { Ministerpräsidentin Ewa Kopacz teilt mit, dass sie nicht an der Gedenkfeier in Smolensk (Russland) zum fünf- } \\
\text { ten Jahrestag der Flugzeugkatastrophe am 10. April teilnehmen, sondern in Warschau auf dem Powazzki-Friedhof } \\
\text { der Todesopfer gedenken wird. Die Delegation, die nach Smolensk fährt, wird von Kulturministerin Małgorzata } \\
\text { Omilanowska und dem Chef der Kanzlei der Ministerpräsidentin, Jacek Cichocki, geleitet. }\end{array}$ \\
\hline 26.03 .2015 & $\begin{array}{l}\text { Staatspräsident Bronisław Komorowski unterzeichnet das vom Sejm und vom Senat verabschiedete Gesetz zur } \\
\text { Zustimmung zur Ratifizierung des Assoziierungsabkommens zwischen der Europäischen Union und Georgien. }\end{array}$ \\
\hline 26.03 .2015 & $\begin{array}{l}\text { Auf Einladung des Bundesratspräsidenten, des hessischen Ministerpräsidenten Volker Bouffier, hält sich eine } \\
\text { Delegation des Senats der Republik Polen unter Leitung von Senatsmarschall Bogdan Borusewicz zu einem zwei- } \\
\text { tägigen Besuch in Hessen und in Berlin auf, wo die Gäste im Hessischen Landtag und im Bundesrat begrüßt } \\
\text { werden und politische Gespräche führen. Zum Auftakt des Besuchs informiert sich die Delegation im Deut- } \\
\text { schen Polen-Institut Darmstadt (DPI) über die Arbeit des Instituts. Der Senatsmarschall trägt sich während sei- } \\
\text { nes Besuchs im DPI auf Einladung von Oberbürgermeister Jochen Partsch in das Goldene Buch der Stadt ein. }\end{array}$ \\
\hline 27.03 .2015 & $\begin{array}{l}\text { Die Vorsitzenden der Polnisch-Russischen Gruppe für schwierige Angelegenheiten, Adam Daniel Rotfeld und } \\
\text { Anatolij Torkunow, beschließen, die für Anfang April terminierte Sitzung zu vertagen. Ein neuer Termin wird } \\
\text { nicht festgelegt. Die gegenwärtige Atmosphäre in den Beziehungen zwischen den Staaten lasse ein Treffen der } \\
\text { Gruppe absurd erscheinen, so Rotfeld. }\end{array}$ \\
\hline
\end{tabular}




\begin{tabular}{|c|c|}
\hline 28.03 .2015 & $\begin{array}{l}\text { Der Kandidat der Polnischen Bauernpartei (Polskie Stronnictwo Ludowe - PSL) für das Amt des Staatsprä- } \\
\text { sidenten, Adam Jarubas, stellt in Opoczno (Woiwodschaft Lodz) sein Programm vor. Prioritär sei für ihn im } \\
\text { Fall eines Wahlsieges die Sicherheit Polens, wozu die Stärkung der polnischen Armee und die Stationierung } \\
\text { von NATO-Basen in Ostpolen gehören. Die Ausrüstung der Armee solle vor allem von polnischen Rüstungs- } \\
\text { unternehmen stammen. Des Weiteren wären die Bekämpfung der demografischen Krise und der Schutz polni- } \\
\text { schen Bodens vor dem Aufkauf durch ausländische Investoren wichtige Aufgabenbereiche seiner Präsidentschaft. }\end{array}$ \\
\hline 30.03 .2015 & $\begin{array}{l}\text { Bei einem Treffen des Weimarer Dreiecks (Deutschland, Frankreich, Polen) in Potsdam fordern die Verteidi- } \\
\text { gungsminister Ursula von der Leyen, Jean-Yves Le Drian und Tomasz Siemoniak einen höheren Stellenwert der } \\
\text { Verteidigungspolitik in der Europäischen Union. In einem Brief an die Hohe Vertreterin der EU für Außen- } \\
\text { und Sicherheitspolitik, Federica Mogherini, stellen sie Reformvorschläge für die Sicherheits- und Außenpoli- } \\
\text { tik der EU vor. }\end{array}$ \\
\hline 31.03 .2015 & $\begin{array}{l}\text { Bei seinem Besuch in Warschau sagt der finnische Staatspräsident Sauli Niinistö Polen Unterstützung im Bereich } \\
\text { der Produktion von Atomenergie zu. }\end{array}$ \\
\hline 01.04 .2015 & $\begin{array}{l}\text { Verteidigungsminister Tomasz Siemoniak und seine niederländische Amtskollegin Jeanine Hennis-Plasschaert } \\
\text { thematisieren in Warschau die Umsetzung der Beschlüsse des NATO-Gipfels in Newport (Wales) im Herbst } \\
\text { vergangenen Jahres. Die dort beschlossene Einrichtung einer superschnellen Eingreiftruppe, die sogenannte } \\
\text { Speerspitze, werde im Juni auf polnischem Territorium erste Übungen durchführen, so Siemoniak. Im kom- } \\
\text { menden Jahr soll die Einheit voll einsatzfähig sein. }\end{array}$ \\
\hline 02.04 .2015 & $\begin{array}{l}\text { Bei ihrem Besuch der polnischen Soldaten und Polizisten im Kosovo, die an den Missionen von KFOR und } \\
\text { EULEX teilnehmen, betont Ministerpräsidentin Ewa Kopacz, dass die Sicherheit eines Landes nicht für immer } \\
\text { gegeben sei. Man müsse sich um sie nicht nur im eigenen Land, sondern auch in den Nachbarländern bemühen. }\end{array}$ \\
\hline 03.04 .2015 & $\begin{array}{l}\text { Die Außenminister des Weimarer Dreiecks (Deutschland, Frankreich, Polen), Frank-Walter Steinmeier, Laurent } \\
\text { Fabius und Grzegorz Schetyna, unterzeichnen in Breslau (Wrockaw) einen Brief an die Hohe Vertreterin der EU } \\
\text { für Außen- und Sicherheitspolitik, Federica Mogherini, in dem sie Prioritäten der EU-Verteidigungspolitik für } \\
\text { die kommenden Jahre und die Felder der Zusammenarbeit in den Bereichen Außenpolitik, Sicherheit und Ver- } \\
\text { teidigung definieren. Der Brief sei mit den Verteidigungsministern des Weimarer Dreiecks abgestimmt worden. }\end{array}$ \\
\hline 03.04 .3015 & $\begin{array}{l}\text { Am Grab des Unbekannten Soldaten in Warschau finden die Hauptfeierlichkeiten zum 75. Jahrestag des Mas- } \\
\text { senmordes in Katyn statt, bei dem mehr als } 20.000 \text { polnische Kriegsgefangene, vor allem Angehörige der pol- } \\
\text { nischen Armee, vom sowjetischen Geheimdienst NKWD getötet worden waren. Anwesend sind neben den } \\
\text { Familien der Opfer Staatspräsident Bronisław Komorowski, Ministerpräsidentin Ewa Kopacz und Verteidi- } \\
\text { gungsminister Tomasz Siemoniak. }\end{array}$ \\
\hline 07.04 .2105 & $\begin{array}{l}\text { In Kattowitz unterzeichnen Vertreter der staatlichen Agentur für die Entwicklung der Industrie (Agencja Rozwoju } \\
\text { Przemysłu - ARP) und der Kokereigruppe JSW Koks eine Absichtserklärung über den Bau eines Heizkraftwerks } \\
\text { in Radlin (Woiwodschaft Schlesien). Produziert werden sollen } 30 \mathrm{MW} \text { Strom und } 100 \mathrm{MW} \text { Wärmeleistung. }\end{array}$ \\
\hline 08.04 .2015 & $\begin{array}{l}\text { Im Rahmen seines zweitägigen Staatsbesuchs in der Ukraine sagt Staatspräsident Bronisław Komorowski dem } \\
\text { Präsidenten der Ukraine, Petro Poroschenko, die Teilnahme Polens an einer eventuellen Friedensmission der } \\
\text { Vereinten Nationen in der Ostukraine zu. }\end{array}$ \\
\hline 09.04 .2015 & $\begin{array}{l}\text { In seiner Rede vor dem ukrainischen Parlament sagt Staatspräsident Bronisław Komorowski, dass sich die } \\
\text { Ukraine faktisch im Kriegszustand befinde und Russland brutal versuche, der Ukraine das Recht auf Selbst- } \\
\text { bestimmung zu nehmen. Die Ukraine stehe vor den schwierigen Herausforderungen, nicht nur das eigene Ter- } \\
\text { ritorium zu verteidigen, sondern auch tiefgehende politische, soziale und wirtschaftliche Reformen durchzu- } \\
\text { führen. Hier müsse die Ukraine Entschlossenheit und der Westen Bereitschaft zur politischen und wirtschaft- } \\
\text { lichen Hilfe sowie auch Geduld zeigen. }\end{array}$ \\
\hline 09.04 .2015 & $\begin{array}{l}\text { Der Untersuchungsausschuss Russlands zum Flugzeugabsturz von Smolensk (Russland), bei dem im Jahr } 2010 \\
96 \text { hochrangige Vertreter des politischen und öffentlichen Lebens Polens tödlich verunglückt waren, teilt mit, } \\
\text { dass Polen das Flugzeugwrack erst nach Abschluss der Ermittlungen zurückgegeben werden könne. Zurzeit } \\
\text { seien noch einige Rechtshilfeersuchen von Polen unbeantwortet geblieben. Die Sprecherin der polnischen Regie- } \\
\text { rung, Małgorzata Kidawa-Błońska, wertet dies als Taktik, die Untersuchungen zu verlängern und die Aushän- } \\
\text { digung des Wracks weiter hinauszuzögern. }\end{array}$ \\
\hline
\end{tabular}




\begin{tabular}{|c|c|}
\hline 10.04 .2015 & $\begin{array}{l}\text { Staatspräsident Bronisław Komorowski, Ministerpräsidentin Ewa Kopacz und weitere politische Repräsentan- } \\
\text { ten nehmen auf dem Powązki-Friedhof in Warschau an der offiziellen Gedenkveranstaltung für die Opfer der } \\
\text { Flugzeugkatastrophe von Smolensk (Russland) vor fünf Jahren teil. Bei dem Flugzeugabsturz waren } 96 \text { hoch- } \\
\text { rangige Vertreter des politischen und öffentlichen Lebens Polens, darunter der damalige Staatspräsident Lech } \\
\text { Kaczyński, tödlich verunglückt. Die Partei Recht und Gerechtigkeit (Prawo i Sprawiedliwość - PiS) gedenkt } \\
\text { der Opfer in Warschau mit einer eigenen Feier. }\end{array}$ \\
\hline 10.04 .2015 & $\begin{array}{l}\text { Eine offizielle Delegation Polens unter der Leitung von Kulturministerin Małgorzata Omilanowska und dem } \\
\text { Leiter der Kanzlei der Ministerpräsidentin, Jacek Cichocki, gedenkt in Smolensk des fünften Jahrestags der } \\
\text { Flugzeugkatastrophe sowie in Katyn des 75. Jahrestags des Massenmordes an polnischen Militärangehörigen } \\
\text { durch den sowjetischen Geheimdienst NKWD. }\end{array}$ \\
\hline 13.04 .2015 & $\begin{array}{l}\text { Nach vorläufigen Schätzungen der Polnischen Nationalbank (Narodowy Bank Polski - NBP) exportierten pol- } \\
\text { nische Firmen im Februar Waren im Wert von 13,7 Mrd. Euro. Dies sind 10,7\% mehr als im Vorjahreszeitraum. }\end{array}$ \\
\hline 14.04 .2015 & $\begin{array}{l}\text { Der Pressesprecher der Demokratischen Linksallianz (Sojusz Lewicy Demokratycznej - SLD), Dariusz Joński, } \\
\text { teilt mit, dass die SLD einen Gesetzesentwurf vorbereitet, der einen Erlass der Einkommensteuer für diejeni- } \\
\text { gen vorsieht, deren Jahreseinkommen maximal } 21.000 \text { Zloty beträgt. Der Entwurf soll im Mai dem Sejm vor- } \\
\text { gelegt werden. }\end{array}$ \\
\hline 15.04 .2015 & $\begin{array}{l}\text { Außenminister Grzegorz Schetyna bewertet die angekündigte Fahrt des russischen Motorradklubs »Nachtwölfe» } \\
\text { durch Polen als politische Provokation. Gleichwohl sei Polen ein Rechtsstaat, den jeder, unter der Bedingung, } \\
\text { das Recht einzuhalten, besuchen könne. Die polnischen Grenzbeamten würden die Entscheidung treffen, ob } \\
\text { auf Seiten der Motorradklubmitglieder die notwendigen Dokumente zur Einreise vorhanden seien. Der russi- } \\
\text { sche Motorradklub plant eine Gedenkfahrt von Moskau nach Berlin aus Anlass des 70. Jahrestages des Sieges } \\
\text { der UdSSR über Deutschland. Einigen Mitgliedern des Motorradklubs wird vorgeworfen, letztes Jahr an der } \\
\text { Annexion der Krim durch Russland aktiv beteiligt gewesen zu sein. }\end{array}$ \\
\hline 16.04 .2015 & $\begin{array}{l}\text { Staatspräsident Bronisław Komorowski und Verteidigungsminister Tomasz Siemoniak besuchen in Lublin das } \\
\text { Hauptquartier der litauisch-polnisch-ukrainischen Militäreinheit LITPOLUKRBRIG. Komorowski unter- } \\
\text { streicht, Polen müsse dafür sorgen, dass die Ukraine Zugang zu den politischen und Sicherheitsstrukturen der } \\
\text { westlichen Welt wie EU und NATO habe. Die internationale Militäreinheit ermögliche der Ukraine die Erfah- } \\
\text { rung von Kooperation und unterstütze den Reformprozess des Verteidigungssystems der Ukraine. Bevor Polen } \\
\text { der NATO beigetreten sei, habe es ähnliche Möglichkeiten im Rahmen des damaligen Programms "Partner- } \\
\text { schaft für den Frieden«nutzen dürfen. }\end{array}$ \\
\hline 17.04 .2015 & $\begin{array}{l}\text { Im Rahmen des III. Strategischen Dialogs Polen - China empfängt Außenminister Grzegorz Schetyna den stell- } \\
\text { vertretenden Außenminister der Volksrepublik China, Wang Chao. Für Polen sei die Entwicklung der strategi- } \\
\text { schen Partnerschaft mit China von großer Bedeutung, so Schetyna. Im Rahmen der wirtschaftlichen Zusam- } \\
\text { menarbeit werden die Perspektiven des Imports von polnischem Schweinefleisch und die Öffnung des chinesi- } \\
\text { schen Markts für polnische Äpfel und andere Produkte der Lebensmittelindustrie thematisiert. }\end{array}$ \\
\hline 18.04 .2015 & $\begin{array}{l}\text { In Dresden findet die achte gemeinsame Sitzung der Präsidien des Bundestages und des Sejm statt. Auf der } \\
\text { Tagesordnung stehen die deutsch-polnischen Beziehungen, die Östliche Partnerschaft, die Perspektiven der } \\
\text { Wirtschafts- und Währungspolitik der EU und die aktuelle Sicherheits- und Verteidigungspolitik der EU ange- } \\
\text { sichts des russisch-ukrainischen Konflikts. }\end{array}$ \\
\hline 19.04 .2015 & $\begin{array}{l}\text { In Warschau wird am Denkmal der Ghettohelden des 72. Jahrestages des Beginns des Ghettoaufstands gegen } \\
\text { die deutsche Besatzung gedacht. Władysław Bartoszewski, ehemaliger Häftling in Auschwitz und ehemaliges } \\
\text { Mitglied des Rates für die Unterstützung der Juden Żegota, Beauftragter der Ministerpräsidentin für den inter- } \\
\text { nationalen Dialog, ruft dazu auf, universal gültige Werte wie die Menschenwürde, die Freiheit des Menschen, } \\
\text { Toleranz, die Fähigkeit zu Freundschaft, Respekt und Opferbereitschaft zu pflegen. }\end{array}$ \\
\hline 20.04 .2015 & $\begin{array}{l}\text { In der tschechischen Hauptstadt Prag finden die polnisch-tschechischen Regierungskonsultationen statt. Thema- } \\
\text { tisiert werden Fragen der Energiesicherheit, der regionalen Zusammenarbeit sowie die Situation in der Ukraine } \\
\text { und in Russland. Ministerpräsidentin Ewa Kopacz wird von Vertretern der Ressorts für Verteidigung, Inne- } \\
\text { res, Infrastruktur und Entwicklung, Umwelt, Landwirtschaft, Wirtschaft und für den Staatsschatz begleitet. }\end{array}$ \\
\hline
\end{tabular}

Sie können die gesamte Chronik seit 2007 auch auf <http://www.laender-analysen.de/polen/> unter dem Link »Chronik«lesen. 
Die Polen-Analysen erscheinen zweimal monatlich als E-Mail-Dienst. Sie werden gemeinsam vom Deutschen PolenInstitut Darmstadt, der Bremer Forschungsstelle Osteuropa und der Deutschen Gesellschaft für Osteuropakunde herausgegeben.

Ein Archiv der Polen-Analysen finden Sie im Internet unter <www.laender-analysen.de/polen>

Kostenloses Abonnement unter < http://www.deutsches-polen-institut.de/Newsletter/subscribe.php>

Diese Analysen finden Sie online als Lizenzausgabe auf

$<$ bpb.de>

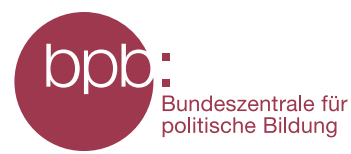

\section{Deutsches Polen-Institut Darmstadt}

Das Deutsche Polen-Institut Darmstadt (DPI) ist ein Forschungs-, Informations-, und Veranstaltungszentrum für polnische Kultur, Geschichte, Politik, Gesellschaft und die deutsch-polnischen Beziehungen, die sich im Kontext der europäischen Integration entwickeln. Das seit März 1980 aktive und bis 1997 von Gründungsdirektor Karl Dedecius geleitete Institut ist eine Gemeinschaftsgründung der Stadt Darmstadt, der Länder Hessen und Rheinland-Pfalz sowie des Bundes. 1987 wurden die Kultusminister der Länder und 2011 das Auswärtige Amt weitere institutionelle Träger. Einen wesentlichen Beitrag zur Verwirklichung der Institutsziele leisten private Stiftungen. Das DPI hat satzungsgemäß die Aufgabe, durch seine Arbeit zur Vertiefung der gegenseitigen Kenntnisse des kulturellen, geistigen und gesellschaftlichen Lebens von Polen und Deutschen beizutragen.

Ziel der Vermittlertätigkeit des DPI ist es, »die zu interessieren, auf die es politisch, wirtschaftlich, gesellschaftlich und kulturell im deutsch-polnischen Verhältnis ankommt (Leitlinien 1997). Es geht um die Entscheider und Multiplikatoren in Politik, Kultur, Bildung, Verwaltung, Medien und Wirtschaft und, wesentlich stärker ausgeprägt als bisher, um das Hineinwirken in Wissenschaft, Forschung und Bildung.

Derzeit bemüht sich das DPI in Kooperation mit den verstreuten Orten wissenschaftlicher Polen-Kompetenz an deutschen Hochschulen und Forschungsinstituten verstärkt darum, ausgehend von einer Bestandsaufnahme deutscher Polen-Forschung Ort wissenschaftlicher Forschung und verbindendes, vernetzendes und kooperierendes Zentrum zu werden. Ausgangspunkt der Neuausrichtung ist die kaum mehr kontrollierbare Dynamik des Rückbaus der Ressourcen der wissenschaftlichen Polen-Kompetenz in den unterschiedlichen Disziplinen. Mit der über 68.000 Bände zählenden multidisziplinären Fachbibliothek für Polen, die eine einzigartige Sammlung polnischer Literatur in der Originalsprache und in deutscher Übersetzung umfasst, ist das DPI bereits ein geschätzter Ort der Recherche und des wissenschaftlichen Arbeitens. (<www.deutsches-polen-institut.de $>$ )

Forschungsstelle Osteuropa an der Universität Bremen (<www.forschungsstelle.uni-bremen.de $>$ ) 1982 gegründet, widmet sich die Forschungsstelle Osteuropa an der Universität Bremen der interdisziplinären Analyse der Länder Ost- und Ostmitteleuropas in Zeitgeschichte und Gegenwart. Der Forschungsschwerpunkt liegt dabei auf der Rolle von "Dissens und Konsens«, von Opposition und Zivilgesellschaft in ihrem historischen, politischen, gesellschaftlichen und kulturellen Kontext. Die Forschungsstelle besitzt in ihrem Archiv eine einzigartige Sammlung alternativer Kulturgüter und unabhängiger Texte aus den ehemaligen sozialistischen Ländern. Darunter befindet sich auch eine umfangreiche Sammlung des "Zweiten Umlaufs«, die das Schrifttum und Dokumente unabhängiger Initiativen und gesellschaftlicher Gruppen in Polen aus der Zeit von 1976 bis zum Umbruch umfasst. Hinzu kommt eine umfangreiche Bibliothek mit wissenschaftlicher Literatur. Mit Archiv, Bibliothek und zwei wissenschaftlichen Abteilungen ist die Forschungsstelle auch eine Anlaufstelle sowohl für Gastwissenschaftler als auch für die interessierte Öffentlichkeit.

Eine der Hauptaufgaben der Forschungsstelle ist die Information der interessierten Öffentlichkeit. Dazu gehören unter anderem regelmäßige E-Mail-Informationsdienste für Politik, Wirtschaft, Zivilgesellschaft und Medien.

Die Meinungen, die in den Polen-Analysen geäußert werden, geben ausschließlich die Auffassung der Autoren wieder. Abdruck und sonstige publizistische Nutzung sind nach Rücksprache mit der Redaktion gestattet. Redaktion: Prof. Dr. Dieter Bingen (verantwortlich) (Darmstadt), Silke Plate, M.A. (Bremen) Technische Gestaltung: Matthias Neumann

Polen-Analysen-Layout: Cengiz Kibaroglu, Matthias Neumann

Alle Ausgaben der Polen-Analysen sind mit Themen- und Autorenindex archiviert unter www.laender-analysen.de

Die Polen-Analysen werden im Rahmen der Datenbank World Affairs Online (WAO) ausgewertet und sind im Portal IREON www.ireon-portal.de recherchierbar.

ISSN 1863-9712 @ 2015 by Deutsches Polen-Institut Darmstadt und Forschungsstelle Osteuropa, Bremen

Kontakt: Dr. Andrzej Kaluza, Presse- und Öffentlichkeitsarbeit, Deutsches Polen-Institut, Mathildenhöhweg 2,

D-64287 Darmstadt, Tel.: 06151/4985-13, Fax: 06151/4985-10, E-Mail: polen-analysen@dpi-da.de, Internet: www.laender-analysen.de/polen 